M. Itô

Nagoya Math. J.

Vol. 57 (1975), 167-197

\title{
GARACTÉRISATION DU PRINCIPE DE DOMINATION POUR LES NOYAUX DE CONVOLUTION NON-BORNÉS
}

\author{
MASAYUKI ITÔ
}

\section{Introduction}

Dans toute la suite $X$ désignera un groupe abélien localement compact, séparé et dénombrable à l'infini; $\xi$ sera sa mesure de Haar. Rappelons qu'un noyau de convolution $N$ sur $X$ signifie une mesure de Radon positive dans $X$ et que, pour une mesure de Radon réelle $\mu$ dans $X, N * \mu$ s'appelle le $N$-potentiel de $\mu$ dès que cette convolution est définie au sens des mesures. Dans cette note, pour simplifier la discussion, nous supposerons qu'il n'existe aucun sous-groupe compact de $X$ excepté $\{0\}$.

On dit qu'un noyau de convolution $N$ sur $X$ est borné si, quelle que soit $f$ une fonction finie et continue dans $X$ à support compact, le $N$-potentiel $N * f$ de $f$ est borné sur $X$. Dans l'article précédent [7], nous avons fournit une caractérisation $d u$ principe de domination pour les noyaux de convolution bornés sur $X$, en utilisant les noyaux de convolution de Hunt. Mais cela n'est pas définitif, car on connaît bien qu'un noyau de convolution sur $X$ est borné et satisfait au principe de domination si et seulement s'il satisfait au principe complet du maximum (cf. par exemple, [5]). On peut donner facilement d'exemples de noyaux de convolution non-bornés et satisfaisant au principe de domination.

Dans cette note, nous founirons une caractérisation définitive du principe de domination pour les noyaux de convolution sur $X$ dans cette direction. Cela est l'énoncé suivant:

Soit $N$ un noyau de convolution sur $X$. Pour que $N$ satisfasse au principe de domination, il faut et il suffit que $N$ soit un noyau de convolution de Hunt sur $X$ ou bien qu'il existe une fonction positive, continue et exponentielle $\varphi$ sur $X$ telle que $N$ soit de la forme

$$
N=\varphi\left(\widetilde{N}_{0}+\widetilde{N}^{\prime}\right)
$$

Received September 17, 1973. 
où $\widetilde{N_{0}}$ est un noyau de convolution borné de Hunt sur $X$ ou bien $\widetilde{N}_{0}=0$ et où $\widetilde{N}^{\prime}$ est un noyau de convolution singulier sur $X$ satisfaisant au principe de domination et périodique à tout le point du support de $\widetilde{N}_{0}$, $\operatorname{supp}\left(\widetilde{N}_{0}\right)$. Dans ce cas, la décomposition de $N$ est essentiellement unique.

\section{Préliminaires}

Dans cet article, on notera toujours

$M_{K}=M_{K}(X ; \xi)$ l'espace vectoriel topologique usuel des fonctions $\xi$-mesurables, bornées dans $X$ à valeurs réelles et à support compact.

$C_{K}=C_{K}(X)$ l'espace vectoriel topologique usuel des fonctions finies et continues dans $X$ à support compact.

$M_{K}^{+}$et $C_{K}^{+}$désignent leur sous-ensembles des fonctions non-négatives.

Rappelons d'abord le principe relatif de domination entre deux noyaux de convolution sur $X$. Soient $N_{1}$ et $N_{2}$ deux noyaux de convolution sur $X$. On dit que $N_{1}$ satisfait au principe de domination relatif à $N_{2}$ si, quelles que soient $f$ et $g$ de $C_{K}^{+}$, l'inégalité $N_{1} * f \leqq N_{2} * g$ est satisfaite partout sur $X$ dès qu'elle l'est sur le support de $f, \operatorname{supp}(f)$. Dans ce cas, on écrit $N_{1} \prec N_{2}$. En particulier, si $N \prec N$, alors on dit simplement que $N$ satisfait au principe de domination.

Remarque 1. Il est un problème si la relation " $\prec$ " est transitive.

D'après l'équivalence de deux types de principes relatifs de domination (cf. [9]) et le résultat dans [8] (cf. le théorème 1 de [8]), le principe relatif du balayage en résulte immédiatement. On dit que $N_{1}$ satisfait au principe du balayage relatif à $N_{2}$ si, pour un ouvert relativement compact $\omega$ de $X$ et pour une mesure de Radon positive $\mu$ dans $X$ à support compact, il existe une mesure de Radon positive $\mu^{\prime}$ dans $X$ portée par $\bar{\omega}$ telle que, au sens des mesures,

(B.1) $N_{1} * \mu_{\omega}^{\prime} \leqq N_{2} * \mu$ dans $X$,

(B.2) $N_{1} * \mu_{\omega}^{\prime}=N_{2} * \mu$ dans $\omega$,

(B.3) Quelle que soit $\nu$ une mesure de Radon positive dans $X$, $N_{1} * \nu \geqq N_{1} * \mu_{\omega}^{\prime}$ dans $X$ dès que $N_{1} * \nu \geqq N_{2} * \mu$ dans $\omega$.

Dans ce cas, $N_{1} * \mu_{\omega}^{\prime}$ est uniquement déterminé et s'appelle le $N_{1}$-potentiel balayé de $N_{2} * \mu$ sur $\omega$. On dit que $\mu_{\omega}^{\prime}$ est une mesure balayée de $\mu$ sur $\omega$ relativement à la couple $\left(N_{1}, N_{2}\right)$. En particulier, si $N=N_{1}$ $=N_{2}, N$ est dit de satisfaire au principe du balayage et $\mu_{\omega}^{\prime}$ s'appelle une mesure balayée de $\mu$ sur $\omega$ relativement au noyau $N$. Si, pour un noyau 
de convolution $N$ sur $X$, le présent énoncé a lieu pour un ouvert quelconque, on dit que $N$ satisfait au principe du balayage sur tout ouvert.

Proposition 1. Soient $N_{1}$ et $N_{2}$ deux noyaux de convolution sur $X$ et supposons que $N_{1} \neq 0$ satisfait au principe de domination. Alors les quatre énoncés suivants sont équivalents:

(a) $N_{1} \prec N_{2}$.

(b) $\check{N}_{1} \prec \check{N}_{2}$.

(c) $N_{1}$ satisfait au principe du balayage relatif à $N_{2}$.

(d) $\check{N}_{1}$ satisfait au principe du balayage relatif $\grave{a} \check{N}_{2}$.

On écrit ici $\check{N}_{i}(i=1,2)$ le noyau de convolution sur $X$ symétrisant avec $N_{i}$ par rapport à l'origine. Voyons l'implication (a) $\Rightarrow(\mathrm{b})$. Supposons que, pour deux fonctions $f, g$ de $C_{K}^{+}, \check{N}_{1} * f \leqq \check{N}_{2} * g$ sur $\operatorname{supp}(f)$; alors $N_{1} * \check{f} \leqq N_{2} * \check{g}$ sur $\operatorname{supp}(\check{f})$, où $\check{f}(x)=f(-x)$, et par suite, d'après $N_{1} \prec N_{2}, N_{1} * \check{f} \leqq N_{2} * \check{g}$ partout sur $X$, d'où $\check{N}_{1} * f \leqq \check{N}_{2} * g$ partout sur $X$. On a donc $\aleph_{1} \prec \aleph_{2}$. Par conséquent, on a l'équivalence (a) $\Leftrightarrow$ (b). On retrouve aussi l'équivalence entre le principe de domination pour $N_{1}$ et le principe de domination pour $\check{N}_{1}$. Donc, en combinant le théorème 1 de [8] et le résultat de [9], on obtient les équivalences (a) $\Leftrightarrow$ (c) et (b) $\Leftrightarrow(d)$, d'où notre proposition.

Proposition 2. Soient $N_{1}$ et $N_{2}$ deux noyaux de convolution sur $X$; supposons que $N_{1} \prec N_{2}$ et $N_{1}$ satisfait au principe de domination. Alors, quelles que soient $f, g$ de $M_{R}^{+}, N_{2} f \leqq N_{2} g$ presque partout pout $\xi$ (noté

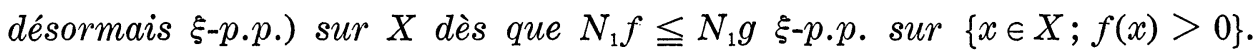

On note ici $N_{1} f$ la densité de la mesure $N_{1} *(f \xi)$ par rapport à $\xi$, qui est localement bornée sur $X$. Voyons notre proposition. D'après la présente proposition, il existe une suite $\left(\varepsilon_{n}^{\prime}\right)_{n=1}^{\infty}$ de mesures de Radon positives dans $X$ telle que $\left(N_{1} * \varepsilon_{n}^{\prime}\right)_{n=1}^{\infty}$ converge d'une manière croissante vers $N_{2}$ avec $n \rightarrow+\infty$. D'après le principe de domination pour $N_{1}$, on a $N_{1} f \leqq N_{1} g \xi$-p.p. sur $X$ (cf. le théorème 1 dans [5]). On a donc

$$
N_{2} *(g \xi)-N_{2} *(f \xi)=\lim _{n \rightarrow+\infty}\left(N_{1} *(g \xi)-N_{1} *(f \xi)\right) * \varepsilon_{n}^{\prime} \geqq 0
$$

au sens des mesures dans $X$, d'où $N_{2} g \geqq N_{2} f \xi$-p.p. sur $X$.

Proposition 3. Soit $N$ un noyau de convolution sur $X$ satisfaisant au principe de domination; alors la totalité $C(N)$ des noyaux de convo- 
lution $N^{\prime}$ sur $X$ vérifiant $N \prec N^{\prime}$ est un cône convexe et vaguement fermé.

En effet, $C(N)$ est évidemment un cône. Soient $N_{1}^{\prime}$ et $N_{2}^{\prime}$ deux noyaux de convolution sur $X$ appartenant à $C(N)$ et $\left(\omega_{n}\right)_{n=1}^{\infty}$ une suite croissante d'ouverts relativement compacts de $X$ avec $\bigcup_{n=1}^{\infty} \omega_{n}=X$. On désigne par $\varepsilon_{n, i}^{\prime}$ une mesure balayée de $\varepsilon$ sur $\omega_{n}$ relativement à $\left(N, N_{i}^{\prime}\right)$, où $\varepsilon$ est la mesure de Dirac à l'origine de $X$. D'après le principe de domination pour $N, N$ satisfait au principe de domination relatif au noyau de convolution $N *\left(\varepsilon_{n, 1}^{\prime}+\varepsilon_{n, 2}^{\prime}\right)$ sur $X$. Faisant $n \rightarrow+\infty$, on arrive à $N \prec N_{1}^{\prime}+N_{2}^{\prime}$, d'où $C(N)$ est un cône convexe. Il est évident que $C(N)$ est vaguement fermé.

Pour un noyau de convolution $N$ sur $X$, on dit qu'une mesure de Radon positive $\eta$ dans $X$ est un $N$-pseudo-potentiel pur s'il existe une famille filtrante $\left(N * \mu_{\alpha}\right)_{\alpha \in \Lambda}$ à droite de $N$-potentiels de mesures de Radon positives dans $X$ telle que l'on ait $\lim _{\alpha \in A} N * \mu_{\alpha}=\eta$ (vaguement). Soit $\eta$ un $N$-pseudo-potentiel pur. Pour un ouvert $\omega$ de $X$, on écrit $\sigma(\eta) \subset \omega$ s'il existe une présente famille filtrante $\left(N * \mu_{\alpha}\right)_{\alpha \in \Lambda}$ à droite telle que $\operatorname{supp}\left(\mu_{\alpha}\right) \subset \omega\left({ }^{\forall} \alpha \in \Lambda\right)$.

Remarque 2. Soit $N$ un noyau de convolution sur $X$ satisfaisant au principe de domination; alors, pour un $N$-pseudo-potentiel pur $\eta$, il existe une suite croissante $\left(N * \mu_{n}\right)_{n=1}^{\infty}$ de $N$-potentiels de mesures de Radon positives dans $X$ qui converge vaguement vers $\eta$ avec $n \rightarrow+\infty$.

En effet, on peut supposer $N \neq 0$. Il existe une famille filtrante $\left(N * \mu_{\alpha}\right)_{\alpha \in \Lambda}$ à droite de $N$-potentiels de mesures de Radon positives dans $X$ qui converge vaguement vers $\eta$. On peut supposer ici que $\mu_{\alpha}$ est à support compact. Soit $\left(\omega_{n}\right)_{n=1}^{\infty}$ une suite croissante d'ouverts relativement compacts avec $\bar{\omega}_{n} \subset \omega_{n+1}$ et $\bigcup_{n=1}^{\infty} \omega_{n}=X$; $\mu_{\alpha, n}^{\prime}$ désigne une mesure balayée de $\mu_{\alpha}$ sur $\omega_{n}$ relativement au noyau $N$. Alors, d'après le principe de domination pour $N,\left(N * \mu_{\alpha, n}^{\prime}\right)_{\alpha \in \Lambda}$ est une famille filtrante à droite dans $X$, et $\left(\mu_{\alpha, n}^{\prime}\right)_{\alpha \in \Lambda}$ est vaguement bornée. Donc il existe une mesure de Radon positive $\mu_{n}^{\prime}$ dans $X$ vérifiant $N * \mu_{n}^{\prime} \leqq \eta$ dans $X$ et $N * \mu_{n}^{\prime}=\eta$ dans $\omega_{n}$. D'après le principe de domination pour $N$ et $\operatorname{supp}\left(\mu_{n}^{\prime}\right) \subset \omega_{n+1},\left(N * \mu_{n}^{\prime}\right)_{n=1}^{\infty}$ est croissante dans $X$, d'où notre remarque.

En généralisant cette remarque, on obtient la proposition suivante.

Proposition 4. Soient $N$ un noyau de convolution sur $X$ satisfaisant au principe de domination et $\eta$ une limite vague d'une famille filtrante 
de $N$-potentiels de mesures de Radon positives dans $X$; alors $\eta$ est une limite vague d'une suite croissante de N-potentiels de mesures de Radon positives dans $X$.

Cela est obtenu, de la même manière que ci-dessus. D'après la proposition 1 et de la même manière que dans le lemme 2 de [7], on obtient la proposition suivante:

Proposition 5. Soient $N_{1}$ et $N_{2}$ deux noyaux de convolution sur $X$; supposons que $N_{1} \prec N_{2}$ et $N_{1}$ satisfait au principe de domination. Alors, pour un $N_{2}$-pseudo-potentiel pur $\eta_{2}$ et pour un ouvert quelconque $\omega$ de $X$, il existe un $N_{1}$-pseudo-potentiel pur $\eta_{1, \omega}$, et un seul tel que, au sens des mesures, $\eta_{1, \omega} \leqq \eta_{2}$ dans $X, \eta_{1, \omega}=\eta_{2}$ dans $\omega$ et $\sigma\left(\eta_{1, \omega}\right) \subset \omega$. En particulier, si $\omega$ est relativement compact, alors $\eta_{1, \omega}$ est un $N_{1}$-potentiel d'une mesure de Radon positive dans $X$.

On dit que $\eta_{1, \omega}$ est le $N_{1}$-pseudo-potentiel balayé de $\eta_{2}$ relativement à $\left(N_{1}, N_{2}\right)$. Le corollaire suivant est un cas spécial de la présente proposition.

Corollaire 1. Soit $N$ un noyau de convolution sur $X$ satisfaisant au principe de domination; alors, pour un voisinage compact $V$ de l'origine, il existe un N-pseudo-potentiel pur $\eta_{C V}$, et un seul tel que, au sens des mesures, $N \geqq \eta_{C V}$ dans $X, N=\eta_{C V}$ dans $C V$ et $\sigma\left(\eta_{C V}\right) \subset C V$.

La définition suivante est déjà introduite dans [7].

DÉfinition 1. Soit $N$ un noyau de convolution sur $X$ satisfaisant au principe de domination; on note

$$
N^{\prime}=\lim _{\substack{V \uparrow X \\ V \in \mathscr{V}_{0}}} \eta_{C V} \quad \text { et } \quad N_{0}=N-N^{\prime}
$$

où $\mathscr{V}_{0}$ est la totalité des voisinages compacts de l'origine. On dit que $N_{0}$ et $N^{\prime}$ sont respectivement la partie régulière et la partie singulière de $N$. En particulier, si $N^{\prime}=0$ (resp. $N_{0}=0$ ), alors $N$ est dit régulier (resp. singulier).

Proposition 6. Soit $N$ un noyau de convolution sur $X$ satisfaisant au principe de domination; alors la partie régulière de $N$ est régulière.

Cela est déjà connu dans le cas où $N$ est borné (cf. le lemme 11 dans [7]). Mais il est essentiel dans sa démonstration que $N$ est borné. 
Démonstration. On peut supposer évidemment $N_{0} \neq 0$ (et donc $N \neq 0$ ). Soient $\mu$ une mesure de Radon positive dans $X$ à support compact et $\omega$ un ouvert relativement compact de $X$. On choisit une suite $\left(\omega_{n}\right)_{n=1}^{\infty}$ d'ouverts relativement compacts de $X$ avec $\bar{\omega}_{n} \subset \omega_{n+1}$ et $\cup_{n=1}^{\infty} \omega_{n}=X$. Pour un voisinage $V$ de $\mathscr{V}_{0}$ avec In $(V) \supset \bar{\omega}$, on note $\omega_{n, C V}=\omega \cup\left(C V \cap \omega_{n}\right)$, où In $(V)$ désigne l'intérieur de $V . N$ satisfaisant au principe du balayage, on désigne par $\mu_{n, C V}^{\prime}$ une mesure balayée de $\mu$ sur $\omega_{n, C V}$ relativement au noyau $N$. Ayant $N \neq 0$, on obtient que $\left(\mu_{n, C V}^{\prime}\right)_{n=1}^{\infty}$ est vaguement bornée, et par suite on peut supposer qu'elle converge vaguement vers une mesure de Radon positive $\mu_{\omega \cup C V}^{\prime}$ dans $X$ avec $n \rightarrow+\infty$. On a $\operatorname{supp}\left(\mu_{\omega \cup C V}^{\prime}\right)$ $\subset \overline{\omega \cup C V}$. La suite $\left(N * \mu_{n, C V}^{\prime}\right)_{n=1}^{\infty}$ converge d'une manière croissante vers un $N$-pseudo-potentiel pur $\eta_{\mu, \omega, C V}$ dans $X$ avec $n \uparrow+\infty$. Pour un voisinage $W$ de $\mathscr{V}_{0}$ avec In $(W) \supset V$, on a

$$
\lim _{n \rightarrow+\infty} \eta_{C W} * \mu_{n, C V}^{\prime}=\eta_{C W} * \mu
$$

au sens des mesures dans In $(W)-V=\{z=x-y ; x \in \operatorname{In}(W), y \in V\}$. On a ensuite

$$
\lim _{n \rightarrow+\infty}\left(N-\eta_{C W}\right) * \mu_{n, C V}^{\prime}=\left(N-\eta_{C W}\right) * \mu_{\omega \cup C V}^{\prime} \leqq N_{0} * \mu_{\omega \cup C V}^{\prime}
$$

au sens des mesures dans $X$, et donc

$$
\eta_{\mu, \omega, C V}-\eta_{C W} * \mu \leqq N_{0} * \mu_{\omega \cup C V}^{\prime}
$$

dans In $(W)-V$. Faisant $W \uparrow X$, on a

$$
\eta_{\mu, \omega, C V}-N^{\prime} * \mu \leqq N_{0} * \mu_{\omega \cup C V}^{\prime}
$$

dans $X$. On a inversement

$$
\eta_{\mu, \omega, C V}-N^{\prime} * \mu=\lim _{n \rightarrow+\infty}\left(N-N^{\prime}\right) * \mu_{n, C V}^{\prime}=\lim _{n \rightarrow+\infty} N_{0} * \mu_{n, C V}^{\prime} \geqq N_{0} * \mu_{\omega \cup C V}^{\prime} .
$$

Par conséquent, on a

$$
\eta_{\mu, \omega, C V}-N^{\prime} * \mu=N_{0} * \mu_{\omega \cup C V}^{\prime},
$$

d'où, au sens des mesures, $N_{0} * \mu \geqq N_{0} * \mu_{\omega \cup C V}^{\prime}$ dans $X$ et $N_{0} * \mu=N_{0} * \mu_{\omega \cup C V}^{\prime}$ dans $\omega$. La famille $\left(\mu_{\omega \cup C V}^{\prime}\right)_{V \in \gamma_{0}, \text { In }(V) \supset \bar{\omega}}$ étant vaguement bornée, on peut supposer qu'elle converge vaguement vers une mesure de Radon positive $\mu_{\omega}^{\prime}$ dans $X$ avec $V \uparrow X$. On a $N_{0} * \mu \geqq N_{0} * \mu_{\omega}^{\prime}$ dans $X$ et $\operatorname{supp}\left(\mu_{\omega}^{\prime}\right) \subset \bar{\omega}$. Soient $\nu$ la restriction de $\mu_{\omega \cup C V}^{\prime}$ sur $\bar{\omega}$ et $\mu_{\omega \cup C V}^{\prime \prime}=\mu_{\omega \cup C V}^{\prime}-\nu$. On désigne 
par $\eta_{\nu, C V}$ le $N$-pseudo-potentiel balayé de $N * \nu$ sur $C V$ relativement au noyau $N$. Alors on a

$$
\eta_{\mu, C V}=\eta_{\mu, \omega, C V}-N * \nu+\eta_{\nu, C V}
$$

dans $X$, où $\eta_{\mu, C V}$ est aussi le $N$-pseudo-potentiel balayé de $N * \mu$ sur $C V$ relativement au noyau $N$. Donc il existe une mesure de Radon positive $\mu_{C V}^{\prime}$ dans $X$ portée par $\overline{C V}$ telle que l'on ait

$$
N * \mu-\eta_{\mu, C V}=N_{0} * \mu-N_{0} * \mu_{C V}^{\prime} \quad \text { et } \quad N_{0} * \mu_{C V}^{\prime} \geqq N_{0} * \mu_{\omega \mathrm{U} C V}^{\prime \prime} \text {. }
$$

On a $\lim _{V \uparrow X} N_{0} * \mu_{C V}^{\prime}=0$, car $N * \mu-\eta_{\mu, C V} \uparrow N_{0} * \mu$ avec $V \uparrow X$. Donc $\left(N_{0} * \mu_{\omega \cup C V}^{\prime \prime}\right)$ converge vaguement vers 0 avec $V \uparrow X$, d'où $N_{0} * \mu=N_{0} * \mu_{\omega}^{\prime}$ au sens des mesures dans $\omega$. Il en résulte que $N_{0}$ satisfait au principe $\mathrm{du}$ balayage ${ }^{1)}$, d'où le principe de domination pour $N_{0}$. Il est facile de voir que $N_{0}$ est régulier, en utilisant l'égalité $N-\eta_{C V}=N_{0}-N_{0} * \varepsilon_{C V}^{\prime}$ pour tout $V$ de $\mathscr{V}_{0}$, où $\varepsilon_{C V}^{\prime}$ est un point adhérent de $\left(\varepsilon_{C V \cap \omega_{n}}^{\prime}\right)_{n=1}^{\infty}$ au sens de la topologie vague. On note ici $\varepsilon_{C V \cap \omega_{n}}^{\prime}$ une mesure balayée de $\varepsilon$ sur $C V \cap \omega_{n}$ relativement au noyau $N$. La démonstration est ainsi complète.

Proposition 7. Soient $N$ un noyau de convolution sur $X$ satisfaisant au principe de domination et $\sigma$ une mesure de Radon positive dans $X$. Si l'on a $N=N * \sigma$, alors $N$ est pseudo-périodique à tout le point $d u$ sous-groupe fermé de $X$ engendré par supp ( $\sigma)$.

On dit que $N$ est périodique (pseudo-périodique) au point $x \in X$ si $N=N * \varepsilon_{x}$ (resp. $N * \varepsilon_{x}$ est propotionnel à $N$ ), où $\varepsilon_{x}$ est la mesure de Dirac au point $x$.

Démonstration. Il est évident que si, pour un point $x$ de $X, N$ est pseudo-périodique à $x$, alors $N$ est pseudo-périodique à $-x$. Si $N$ $=N * \sigma$, alors pour tout l'entier $n>0, N *(\sigma)^{n}=N$, où $(\sigma)^{0}=\varepsilon$ et $(\sigma)^{n}$ $=(\sigma)^{n-1} * \sigma$. Donc pour notre proposition, il suffit de voir que $N$ est pseudo-périodique à tout le point de $\operatorname{supp}(\sigma)$. On peut supposer $N \neq 0$, et donc $\sigma \neq 0$. Soient $x$ un point quelconque de $\operatorname{supp}(\sigma)$ et $V(x)$ un voisinage ouvert et relativement compact de $x$. On désigne par $\varepsilon_{V(x)}^{\prime}$ une mesure balayée de $\varepsilon$ sur $V(x)$ relativement au noyau $N$. On a alors

1) $\mathrm{Si}$, pour une mesure de Radon positive $\mu$ dans $X$ à support compact et pour un ouvert relativement compact $\omega$, il existe une mesure de Radon positive dans $X$ portée par $\bar{\omega}$ et vérifiant (B.1) et (B.2) pour $N_{0}=N_{1}=N_{2}$, on peut voir facilement l'existence d'une mesure de Radon positive dans $X$ portée par $\bar{\omega}$ et verifiant (B.1), (B.2) et (B.3). 


$$
N *\left(\varepsilon-\varepsilon_{V(x)}^{\prime}\right) * \sigma=N *\left(\varepsilon-\varepsilon_{V(x)}^{\prime}\right)
$$

dans $X . \quad$ Si $\operatorname{supp}\left(N *\left(\varepsilon-\varepsilon_{V(x)}^{\prime}\right)\right) \ni 0$, alors $\operatorname{supp}\left(N *\left(\varepsilon-\varepsilon_{V(x)}^{\prime}\right) * \sigma\right) \ni x$. Mais cela est en contradiction avec $N=N * \varepsilon_{V(x)}^{\prime}$ dans $V(x)$. Donc $N=N * \varepsilon_{V(x)}^{\prime}$ dans un voisinage ouvert de l'origine. D'après le principe de domination pour $N$, on a $N \leqq N * \varepsilon_{V(x)}^{\prime}$ dans $X$, d'où $N=N * \varepsilon_{V(x)}^{\prime}$ dans $X$. Ayant $N \neq 0$, on obtient que $\left(\varepsilon_{V(x)}^{\prime}\right)$ est vaguement bornée lorsque $V(x)$ tend d'une manière décroissante vers $\{x\}$. Par conséquent, il existe une constante $c_{x}>0$ tel que $N * \varepsilon_{x}=c_{x} N$, d'où notre proposition.

DÉFINITION 2. Soit $\varphi$ une fonction définie sur $X$. Si, quels que soient $x$ et $y$ de $X, \varphi(x+y)=\varphi(x) \varphi(y)$, alors $\varphi$ est dite exponentielle.

Remarque 3. Soit $X^{\prime}$ un sous-groupe fermé de $X$. Il est élémentaire qu'une fonction positive, continue et exponentielle sur $X^{\prime}$ peut être prolongée à une fonction positive, continue et exponentielle sur $X$. Mais ce prolongement n'est pas toujours unique.

Enonçons qu'un noyau de convolution régulier est contenu dans un bonne classe de noyaux dans la théorie du potentiel. On dit qu'un noyau de convolution $N$ sur $X$ est un noyau de convolution de Hunt sur $X$ s'il existe un semi-groupe vaguement continu $\left(\alpha_{t}\right)_{t \geqq 0}$ de mesures de Radon positives dans $X$ avec $\alpha_{0}=\varepsilon$ tel que l'on ait $N=\int_{0}^{\infty} \alpha_{t} d t$. Dans ce cas, $\left(\alpha_{t}\right)_{t \geqq 0}$ est uniquement déterminé (cf. par example, [3]) et s'appelle le semi-groupe associé au noyau $N$. Cela est un noyau de convolution possédant les propriétés définitives dans la théorie du potentiel.

Proposition 8. Un noyau de convolution $N$ sur $X$ est régulier si et seulement si $N=0$ ou bien $N$ est un noyau de convolution de Hunt sur $X$.

Pour montrer la proposition 9, on préparera d'abord les trois lemmes suivants. Le lemme 1 est un théorème de J. Deny (cf. [3]).

LEMme 1. Soit $N$ un noyau de convolution sur $X$; alors pour que $N$ soit un noyau de convolution de Hunt sur $X$, il faut et if suffit que $N$ soit un noyau de convolution associé sur $X$, qui est, par définition, un noyau de convolution sur $X$ vérifiant la condition suivante:

Il existe une base de voisinages compacts $\mathscr{V}$ de l'origine telle que l'on puisse associer à tout l'élément $V$ de $\mathscr{V}$ une mesure de Radon positive $\sigma_{V}$ dans $X$ vérifiant les trois conditions suivantes: 
(A.1) $N \geqq N * \sigma_{V}$ dans $X$ et $N \neq N * \sigma_{V}$.

(A.2) $N=N * \sigma_{V}$ dans $C V$.

(A.3) $N *\left(\sigma_{V}\right)^{n}$ converge vaguement vers 0 avec $n \rightarrow+\infty$.

Lemme 2. Soient $N$ un noyau de convolution $(\neq 0)$ sur $X$ et $X^{\prime}$ un sous-groupe fermé de $X$. Si $N$ est pseudo-périodique à tout le point de $X^{\prime}$, alors il existe une fonction positive, continue et exponentielle $\varphi$ sur $X$ telle que $\varphi N$ soit périodique à tout le point de $X^{\prime}$, où $\varphi N$ signifie une mesure de Radon positive dans $X$ dont la densité par rapport à $N$ soit égale à $\varphi$.

En effet, pour un point $x$ de $X^{\prime}$, il existe une constante $c_{x}>0$ telle que $N * \varepsilon_{x}=c_{x} N$. Posons $\varphi(x)=c_{x}$ pour tout $x$ de $X^{\prime}$; alors $\varphi$ est évidemment une fonction exponentielle sur $X^{\prime}$, et elle est continue, car $N * \varepsilon_{x} \rightarrow N * \varepsilon_{x_{0}}$ (vaguement) avec $x \rightarrow x_{0}\left(x, x_{0} \in X^{\prime}\right)$. Donc on peut supposer que $\varphi$ est une fonction positive, continue et exponentielle sur $X$. On a, pour tout $x$ de $X^{\prime}$,

$$
(\varphi N) * \varepsilon_{x}=\frac{\varphi}{\varphi(x)}\left(N * \varepsilon_{x}\right)=\varphi N,
$$

d'où $\varphi N$ est périodique à tout le point de $X^{\prime}$.

LEMme 3. Soient $N$ un noyau de convolution sur $X$ satisfaisant au principe de domination et $N_{0}, N^{\prime}$ sa partie régulière, sa partie singulière, respectivement. Alors pour toute la fonction positive, continue et exponentielle $\varphi$ sur $X, \varphi N$ satisfait au principe de domination, et sa partie régulière et sa partie singulière sont respectivement égaux $\grave{a} \varphi N_{0}$ et $\grave{a}$ $\varphi N^{\prime}$.

En effet, supposons que, pour deux fonctions $f$ et $g$ de $C_{K}^{+}$,

$$
(\varphi N) * f \leqq(\varphi N) * g \quad \text { sur } \quad \operatorname{supp}(f) ;
$$

posons $f^{\prime}=f / \varphi$ et $g^{\prime}=g / \varphi$. Alors on a $\varphi\left(N * f^{\prime}\right) \leqq \varphi\left(N * g^{\prime}\right)$ sur supp $(f)$ $=\operatorname{supp}\left(f^{\prime}\right)$. D'après le principe de domination pour $N$, on a $\varphi\left(N * f^{\prime}\right)$ $\leqq \varphi\left(N * g^{\prime}\right)$ sur $X$, d'où le principe de domination pour $\varphi N$. Pour la deuxième part de ce lemme, il suffit que, quel que soit $V$ de $\mathscr{V}_{0}$, le $(\varphi N)$-pseudo-potentiel balayé de $\varphi N$ sur $C V$ relativement au noyau $\varphi N$ soit égal à $\varphi \eta_{C V}$, où $\eta_{C V}$ est le $N$-pseudo-potentiel balayé de $N$ sur $C V$ relativement au noyau $N$. Pour un ouvert relativement compact $\omega$ de 
$X$, on désigne par $\varepsilon_{\omega}^{\prime}$ une mesure balayée de $\varepsilon$ sur $\omega$ relativement au noyau $N$. Alors on a

$$
(\varphi N) *\left(\varphi \varepsilon_{\omega}^{\prime}\right)=\varphi\left(N * \varepsilon_{\omega}^{\prime}\right),
$$

et donc $\varphi \varepsilon_{\omega}^{\prime}$ est une mesure balayée de $\varepsilon$ sur $\omega$ relativement au noyau $\varphi N$. Donc le présent énoncé en résulte immédiatement.

Démonstration de la proposition 8. Il est connu que la part de "si" a lieu (cf. par exemple, [7]). Donc on montrera son inverse. On peut supposer $N \neq 0$. Dans la démonstration de la proposition 6, on obtient pour un voisinage $V$ de $\mathscr{V}_{0}$, il existe une mesure de Radon positive $\varepsilon_{C V}^{\prime}$ dans $X$ portée par $\overline{C V}$ telle que

$$
N-\eta_{C V}=N_{0}-N_{0} * \varepsilon_{C V}^{\prime},
$$

où $\eta_{C V}$ est le $N$-pseudo-potentiel balayé de $N$ sur $C V$ relativement au noyau $N$. Ayant $N=N_{0}$, on a $\eta_{C V}=N * \varepsilon_{C V}^{\prime}$, et par suite $N \geqq N * \varepsilon_{C V}^{\prime}$ dans $X$ et $N=N * \varepsilon_{C V}^{\prime}$ dans $C V$. Supposons qu'il existe un voisinage $V$ de $\mathscr{V}_{0}$ tel que $N=N * \varepsilon_{C V}^{\prime}$ dans $X$. On a $\varepsilon_{C V}^{\prime} \neq 0$, car $N \neq 0$. Alors, d'après la proposition 7 et le lemme 2, il existe une fonction positive, continue et exponentielle $\varphi$ sur $X$ telle que $\varphi N$ soit périodique à tout le point du sous-groupe fermé $X^{\prime}$ de $X$ engendré par $\operatorname{supp}\left(\varepsilon_{C V}^{\prime}\right)$. D'après notre hypothèse concernant $X$ et $\operatorname{supp}\left(\varepsilon_{C V}^{\prime}\right) \not \supset 0, X^{\prime}$ est non-compact. Alors pour tout le voisinage $U$ de $\mathscr{V}_{0}$, il existe une mesure de Radon positive $\varepsilon_{C U}^{\prime \prime}$ dans $X$ avec $\operatorname{supp}\left(\varepsilon_{C V}^{\prime \prime}\right) \subset C U$ telle que $(\varphi N)=(\varphi N) * \varepsilon_{C U}^{\prime \prime}$ dans $X$. D'après le principe de domination pour $\varphi N$, on a

$$
\varphi\left(N * \varepsilon_{C U}^{\prime}\right)=(\varphi N) *\left(\varphi \varepsilon_{C U}^{\prime}\right) \geqq(\varphi N) * \varepsilon_{C U}^{\prime \prime}
$$

dans $X$, et donc $\varphi N$ n'est pas régulier. Mais cela est en contradiction avec le lemme 3. Par conséquent, on a $N \neq N * \varepsilon_{C V}^{\prime}$ pour tout $V$ de $\mathscr{V}_{0}$. Montrons finalement que, pour tout $V$ de $\mathscr{V}_{0}, N *\left(\varepsilon_{C V}^{\prime}\right)^{n}$ converge vaguement vers 0 avec $n \rightarrow+\infty$. La suite $\left(N *\left(\varepsilon_{C V}^{\prime}\right)^{n}\right)_{n=1}^{\infty}$ est évidemment décroissante, et on désigne par $\eta$ sa limite vague. On a $\eta=\eta * \varepsilon_{C V}^{\prime}$ et $\eta \geqq N$. Pour un entier $m \geqq 1$ quelconque, on a

$$
\left(\sum_{n=0}^{m}\left(\varepsilon_{C V}^{\prime}\right)^{n}\right) *\left(N *\left(\varepsilon-\varepsilon_{C V}^{\prime}\right)\right)=N-N *\left(\varepsilon_{C V}^{\prime}\right)^{m+1} \leqq N,
$$

et donc $\sum_{n=0}^{\infty}\left(\varepsilon_{C V}^{\prime}\right)^{n}$ a un sens, d'où $\left(\varepsilon_{C V}^{\prime}\right)^{n}$ converge vaguement vers 0 avec $n \rightarrow+\infty$. Donc, pour tout $U$ de $\mathscr{V}_{0},\left(N *\left(\varepsilon-\varepsilon_{C U}^{\prime}\right)\right) *\left(\varepsilon_{C V}^{\prime}\right)^{n}$ converge 
vaguement vers 0 avec $n \rightarrow+\infty$, et par suite $\eta=\eta * \varepsilon_{C V}^{\prime}$. Par conséquent, on a $\eta \leqq N * \varepsilon_{C U}^{\prime}$, et par suite, en faisant $U \uparrow X, \eta=0$. D'après le lemme $1, N$ est un noyau de convolution de Hunt sur $X$. La démonstration est ainsi complète.

CoRollaIRE 2. Soit $N$ un noyau de convolution sur $X$ satisfaisant au principe de domination. Pour que $N$ satisfasse au principe d'unicité, il faut et il suffit que sa partie régulière ne s'annule pas.

Dans la théorie générale (cf. par exemple, [3]), les noyaux de Hunt satisfont au principe d'unicité, et par suite il en résulte que la condition est suffisante. La condition est évidemment nécessaire. On rappelle que $N$ satisfait au principe d'unicité si, quelles que soient $\mu$ et $\nu$ de mesures de Radon positives dans $X, \mu=\nu$ dès que $N * \mu=N * \nu$.

CoRollaIre 3. Soit $N$ un noyau de convolution sur $X$ satisfait au principe de domination. Si la partie régulière de $N$ ne s'annule pas, alors, pour une mesure de Radon positive $\mu$ dans $X$ à support compact et pour un ouvert relativement compact $\omega$ de $X$, une mesure balayée de $\mu$ sur $\omega$ relativement au noyau $N$ est uniquement déterminée.

En effet, soient $\mu^{\prime}$ et $\mu^{\prime \prime}$ deux mesures balayées de $\mu$ sur $\omega$ relativement au noyau $N$; alors $N * \mu^{\prime}=N * \mu^{\prime \prime}$, et donc $\mu^{\prime}=\mu^{\prime \prime}$.

Dans ce cas, $\mu^{\prime}$ est dite la mesure balayée de $\mu$ sur $\omega$ relativement au noyau $N$.

\section{Le théorème principal}

Ce paragraphe sera toujours consacrée à la démonstration du théorème suivant:

THÉORÈme. Soit $N$ un noyau de convolution sur $X$. Alors pour que $N$ satisfasse au principe de domination, il faut et il suffit que $N$ soit un noyau de convolution de Hunt sur $X$ ou bien qu'il existe une fonction $\varphi$ positive, continue et exponentielle sur $X$ telle que

$$
N=\varphi\left(\widetilde{N}_{0}+\widetilde{N}^{\prime}\right),
$$

où $\widetilde{N}_{0}$ est un noyau de convolution borné de Hunt sur $X$ ou bien $\widetilde{N}_{0}=0$ et où $\widetilde{N}^{\prime}$ est un noyau de convolution singulier $(\neq 0)$ sur $X$ (satisfaisant au principe de domination) périodique à tout le point de $\operatorname{supp}\left(N_{0}\right) . \quad$ Dans ce cas, $\varphi$ est uniquement déterminée sur le sous-groupe fermé engendré 
par le support de la partie régulière de $N$, et la décomposition de $N$ est unique.

Si ce théorème a lieu, alors le théorème de [7] en résulte immédiatement, car si $N$ est borné, alors $\varphi=1$. On va préparer les quelques lemmes suivants.

Lemme 4. Soit $N$ un noyau de convolution sur $X$; alors il existe une fonction finie, continue et strictement positive dans $X$ telle que $N * f$ soit fini et continu.

En effet, soit $\left(\omega_{n}\right)_{n=1}^{\infty}$ une suite d'ouverts relativement compacts telle que $X=\bigcup_{n=1}^{\infty} \omega_{n}$ et $\bar{\omega}_{n} \subset \omega_{n+1}$. On choisit une fonction $f_{n}$ de $C_{K}^{+}$telle que $0 \leqq f_{n} \leqq 1, f_{n}=1$ sur $\bar{\omega}_{n}$ et $\operatorname{supp}\left(f_{n}\right) \subset \omega_{n+1}$. On choisit une constante $a_{n}$ telle que $0<a_{n} \leqq 2^{-n}$ et $a_{n} N * f_{n}<2^{-n}$ sur $\bar{\omega}_{n}$. Posons $f=\sum_{n=1}^{\infty} a_{n} f_{n}$; alors $f$ est une fonction que nous avons désiré.

LEMME 5. Soient $N$ un noyau de convolution sur $X$ satisfaisant au principe de domination et $\eta$ une limite d'une suite croissante $\left(N * \mu_{n}\right)_{n=1}^{\infty}$ de $N$-potentiels de mesures de Radon positives dans $X$. Alors, quels que soient $\eta^{\prime}$ un $N$-pseudo-potentiel pur et $\left(N * \nu_{n}\right)_{n=1}^{\infty}$ une suite croissante tendant vers $\eta^{\prime}$, on $a$

$$
\lim _{n \rightarrow+\infty} \eta * \nu_{n}=\lim _{n \rightarrow+\infty} \eta^{\prime} * \mu_{n} \text { (vaguement) }
$$

Cela résulte $\mathrm{du}$ fait que

$$
N * \mu_{n_{1}} * \nu_{m_{1}} \leqq N * \mu_{n_{2}} * \nu_{m_{2}}
$$

dès que $n_{1} \leqq n_{2}$ et $m_{1} \leqq m_{2}$.

DÉFINITION 3. Soient $N$ un noyau de convolution sur $X$ satisfaisant au principe de domination et $\eta$ une mesure de Radon réelle dans $X$. On dit que $\eta$ est $N$-invariante si, pour tout $V$ de $\mathscr{V}_{0}$, il existe une suite d'ouverts relativement compacts $\left(\omega_{n}\right)_{n=1}^{\infty}$ de $X$ avec $\bar{\omega}_{n} \subset \omega_{n+1}$ et $\bigcup_{n=1}^{\infty} \omega_{n}=X$ tell que $\eta * \varepsilon_{C V \cap \omega_{n}}^{\prime}$ converge vaguement vers $\eta$ avec $n \rightarrow+\infty$, où $\varepsilon_{C V \cap \omega_{n}}^{\prime}$ est une mesure balayée de $\varepsilon$ sur $C V \cap \omega_{n}$ relativement au noyau $N$. En particulier, si $N$ est un noyau de convolution de Hunt, $\eta$ est dite $N$ harmonique.

Remarque 4. Soit $\left(\omega_{n}\right)_{n=1}^{\infty}$ une suite d'ouvert relativement compacts de $X$ telle qne $\bar{\omega}_{n} \subset \omega_{n+1}$ et $\bigcup_{n=1}^{\infty} \omega_{n}=X$. Pour qu'un $N$-pseudo-potentiel 
pur $\eta$ soit $N$-invariant, il faut et il suffit que l'on ait $\lim _{n \rightarrow+\infty} \eta * \varepsilon_{C V \cap \omega_{n}}=\eta$ (vaguement).

Cela résulte du lemme 5 .

LEMME 6. Soit $N$ un noyau de convolution sur $X$ satisfaisant au principe de domination et dont la partie régulière ne s'annule pas. Pour qu'un autre noyau de convolution $N_{1}$ sur $X$ vérifie $N \prec N_{1}$, il faut et il suffit que $N_{1}$ soit de la forme

$$
N_{1}=N * \mu+\eta,
$$

où $\mu$ est une mesure de Radon positive dans $X$ et $\eta$ est un N-pseudopotentiel pur et $N$-invarint. Dans ce cas, la décomposition de $N_{1}$ est unique.

En effet, soit $\left(\omega_{n}\right)_{n=1}^{\infty}$ une suite d'ouverts relativement compacts de $X$ avec $\bar{\omega}_{n} \subset \omega_{n+1}$ et $\bigcup_{n=1}^{\infty} \omega_{n}=X$; supposons $N \prec N_{1}$. D'après la proposition $1, N$ satisfait au principe du balayage relatif à $N_{1}$, et donc on désigne par $\mu_{n}^{\prime}$ une mesure balayée de $\varepsilon$ sur $\omega_{n}$ relativement à $\left(N, N_{1}\right)$. De la même manière que dans le corollaire $3, \mu_{n}^{\prime}$ est uniquement déterminée, et donc $\mu_{n}^{\prime} \geqq \mu_{n+1}^{\prime}$ au sens des mesures dans $\omega_{n}$, car $\mu_{n}^{\prime}$ est aussi une mesure balayée de $\mu_{n+1}^{\prime}$ sur $\omega_{n}$ relativement au noyau $N$. On a $\lim N * \mu_{n}^{\prime}=N_{1}$ (vaguement). Ayant $N \neq 0$ et $N * \mu_{n}^{\prime} \leqq N_{1}$, on obtient que $\left(\mu_{n}^{\prime}\right)_{n=1}^{\infty}$ est vaguement bornée, et par suite $\left(\mu_{n}^{\prime}\right)_{n=1}^{\infty}$ converge vaguement vers une mesure de Radon positive $\mu$ dans $X$ avec $n \rightarrow+\infty$. Posons $\eta=N_{1}-N * \mu$; alors on a

$$
\eta=\lim _{n \rightarrow+\infty} N *\left(\mu_{n}^{\prime}-\mu_{n}\right) \text { (vaguement) }
$$

où $\mu_{n}$ est la restreinte de $\mu$ sur $\omega_{n}$. La mesure $\mu_{n}^{\prime}-\mu_{n}$ étant positive, $\eta$ est un $N$-pseudo-potentiel pur (cf. la proposition 4). Soit $V$ un voisinage de $\mathscr{V}_{0}$; on désigne par $\varepsilon_{n}^{\prime}$ la mesure balayée de $\varepsilon$ sur $C V \cap \omega_{n}$ relativement au noyau $N$. Alors $\left(\eta * \varepsilon_{n}^{\prime}\right)_{n=1}$ est croissante et on a $\eta \geqq \eta * \varepsilon_{n}^{\prime}$. On a, d'après le lemme 5 ,

$$
\lim _{m \rightarrow+\infty}\left(\lim _{n \rightarrow+\infty} N * \mu_{n}^{\prime}\right) * \varepsilon_{m}^{\prime}=\lim _{n \rightarrow+\infty} \eta_{C V} * \mu_{n}^{\prime} \text { (vaguement), }
$$

où $\eta_{C V}$ est le $N$-pseudo-potentiel balayé de $N$ sur $C V$ relativement au noyau $N$. On a donc 


$$
\begin{aligned}
\lim _{m \rightarrow+\infty} \eta *\left(\varepsilon-\varepsilon_{n}^{\prime}\right) & =\lim _{m \rightarrow+\infty}\left(\lim _{n \rightarrow+\infty} N * \mu_{n}^{\prime} *\left(\varepsilon-\varepsilon_{n}^{\prime}\right)-N * \mu *\left(\varepsilon-\varepsilon_{n}^{\prime}\right)\right) \\
& =\lim _{n \rightarrow+\infty}\left(N-\eta_{C V}\right) *\left(\mu_{n}^{\prime}-\mu\right)=0,
\end{aligned}
$$

d'où $\eta$ est $N$-invariant. Voyons l'unicité de cette décomposition. Soit $N=N * \mu^{\prime}+\eta^{\prime}$ une autre décomposition de $N$. Alors, en utilisant le lemme 5 , on a, pour tout $V$ de $\mathscr{V}_{0}$,

$$
\left(N-\eta_{C V}\right) * \mu=\left(N-\eta_{C V}\right) * \mu^{\prime}
$$

dans $X$, et par suite, faisant $V \uparrow X$, on a $N_{0} * \mu=N_{0} * \mu^{\prime}$. D'après les propositions 6 et 7 , on a $\mu=\mu^{\prime}$, et donc $\eta=\eta^{\prime}$. L'inverse a évidemment lieu, d'où notre lemme.

D'après le présent lemme, tout le $N^{\prime}$-pseudo-potentiel pur est $N$ invariant, où $N^{\prime}$ est la partie singulière de $N$. On remarque ici que tout le $N^{\prime}$-pseudo-potentiel pur est un $N$-pseudo-potentiel pur.

Jusqu'à la démonstration du théorème, $N$ est toujours un noyau de convolution sur $X$ satisfaisant au principe de domination et dont la partie régulière ne s'annule pas, et on note $N_{0}$ et $N^{\prime}$ la partie régulière de $N$ et la partie singulière de $N$, respectivement. Pour une fonction positive, finie et continue $f$ dans $X$ telle que $N * f$ soit fini et continu, on note

$C\left(N^{\prime} ; f\right)=\left\{N_{1}\right.$ : noyau de convolution sur $\left.X ; N \prec N_{1}, N_{1} * f(0) \leqq N^{\prime} * f(0)\right\}$.

LEMME 7. $C\left(N^{\prime} ; f\right)$ est un ensemble convexe et vaguement compact.

En effet, $C\left(N^{\prime} ; f\right)$ est évidemment convexe. D'après $f>0$ sur $X$ et $N_{1} * f(0) \leqq N^{\prime} * f(0)\left(\forall N_{1} \in C\left(N^{\prime} ; f\right)\right), C\left(N^{\prime} ; f\right)$ est relativement compact Soit $N_{1}$ un élément adhérent de $C\left(N^{\prime} ; f\right)$; alors, d'après la proposition $4, N_{1}$ est un $N$-pseudo-potentiel pur, et par suite $N \prec N_{1}$, d'où notre lemme.

On désigne par Ex. $C\left(N^{\prime} ; f\right)$ l'ensemble constitué par tous les éléments extrêmes. On se propose de déterminer explicitement Ex. $C\left(N^{\prime} ; f\right)$.

LEMme 8. Soit $N_{1}$ un noyau de convolution sur $X$. Alors pour que $N_{1}$ appartienne à Ex. $C\left(N^{\prime} ; f\right)$, il faut et il suffit que $N_{1}=0$,

$$
N_{1}=\frac{N^{\prime} * f(0)}{N * f(-x)} N * \varepsilon_{x},
$$

où $x$ est un point de $X$,ou bien que $N_{1}$ soit un $N$-pseudo-potentiel pur, 
$N$-invariant, avec $N_{1} * f(0)=N^{\prime} * f(0)$ et vérifiant la condition suivante:

Quel que soit $\eta$ un N-pseudo-potentiel pur tel que $N_{1}-\eta$ le soit aussi, on $a \eta=c N_{1}$, où $c$ est une constante avec $0 \leqq c \leqq 1$.

En effet, on peut supposer $N^{\prime} \neq 0$. Montrons que la condition est nécessaire. Tout l'élément $N_{1}$ de Ex. $C\left(N^{\prime} ; f\right)$ vérifie évidemment $N_{1} * f(0)$ $=N^{\prime} * f(0)$ dès que $N_{1} \neq 0$. D'après le lemme $6, N_{1}$ est un $N$-potentiel d'une mesure de Radon positive dans $X$ ou bien un $N$-pseudo-potentiel pur et $N$-invariant. Si $N_{1}$ est le premier, alors il existe un point $x$ de $X$ telle que

$$
N_{1}=\frac{N^{\prime} * f(0)}{N * f(-x)} N * \varepsilon_{x}
$$

car, quelle que soit $\mu$ une mesure de Radon positive dans $X, N * \mu$ $=\int N * \varepsilon_{x} d \mu(x)$. Soient $N_{1}$ le dernier et $\eta$ un $N$-pseudo-potentiel pur $(\neq 0)$ tel que $N_{1}-\eta$ le soit aussi. On a $\eta * f(0)>0$ et

$$
N_{1} \pm\left(N_{1}-\frac{N_{1} * f(0)}{\eta * f(0)} \eta\right) \frac{\eta * f(0)}{N_{1} * f(0)} \in C\left(N^{\prime} ; f\right)
$$

et donc

$$
N_{1}=\frac{N_{1} * f(0)}{\eta * f(0)} \eta
$$

Par conséquent, la condition est nécessaire. Montrons que la condition est suffisante. Evidemment $0 \in \operatorname{Ex} . C\left(N^{\prime} ; f\right)$. Soient $x$ un point de $X$ et $N_{1}=\left(N^{\prime} * f(0) / N * f(-x)\right) N * \varepsilon_{x}$; supposons que, pour deux noyaux de convolution $N_{1}^{\prime}, N_{1}^{\prime \prime}$ sur $X$ appartenant à $C\left(N^{\prime} ; f\right)$ et pour une constante $\alpha$ avec $0<\alpha<1, N_{1}=\alpha N_{1}^{\prime}+(1-\alpha) N_{1}^{\prime \prime}$. D'après le lemme 6 , il existe deux mesures de Radon positives $\mu^{\prime}$ et $\mu^{\prime \prime}$ dans $X$ telles que, quel que soit $V$ de $\mathscr{V}_{0}$,

$$
\frac{N^{\prime} * f(0)}{N * f(-x)}\left(N-\eta_{C V}\right) * \varepsilon_{x}=\alpha\left(N-\eta_{C V}\right) * \mu^{\prime}+(1-\alpha)\left(N-\eta_{C V}\right) * \mu^{\prime \prime},
$$

et par suite, faisant $V \uparrow X$, on a

$$
N_{0} *\left(\frac{N^{\prime} * f(0)}{N * f(-x)} \varepsilon_{x}\right)=N_{0} *\left(\alpha \mu^{\prime}+(1-\alpha) \mu^{\prime \prime}\right) .
$$

D'après le principe d'unicité pour $N_{0}$, on a 


$$
\frac{N^{\prime} * f(0)}{N * f(-x)} \varepsilon_{x}=\alpha \mu^{\prime}+(1-\alpha) \mu^{\prime \prime}
$$

On a donc $\mu^{\prime}=a_{1} \varepsilon_{x}$ et $\mu^{\prime \prime}=a_{2} \varepsilon_{x}$, où $a_{i}(i=1,2)$ est une constante $\geqq 0$. $N * \varepsilon_{x}$ n'est pas $N$-invariant, et donc on a

$$
N_{1}^{\prime}=a_{1} N * \varepsilon_{x} \quad \text { et } \quad N_{1}^{\prime \prime}=a_{2} N * \varepsilon_{x} \text {. }
$$

D'après $N^{\prime} * f(0)=N_{1}^{\prime} * f(0)=N_{1}^{\prime \prime} * f(0)$, on a $a_{1}=a_{2}=N^{\prime} * f(0) / N * f(-x)$, d'où $N_{1}=N_{1}^{\prime}=N_{1}^{\prime \prime}$. Par conséquent, $N_{1} \in \operatorname{Ex} . C\left(N^{\prime} ; f\right)$. D'autre part, soit $N_{1}$ un noyau de convolution sur $X$ vérifiant la dernière condition. Si, pour deux noyaux de convolution $N_{1}^{\prime}, N_{1}^{\prime \prime}$ sur $X$ appartenant à $C\left(N^{\prime} ; f\right)$ et pour $0<\alpha<1, N_{1}=\alpha N_{1}^{\prime}+(1-\alpha) N_{1}^{\prime \prime}$, alors, d'après notre condition, on a $\alpha N_{1}^{\prime}=c_{1} N_{1}$ et $(1-\alpha) N_{1}^{\prime \prime}=c_{2} N_{1}$, où $c_{i}(i=1,2)$ est une constante $\geqq 0$. D'après $N_{1}^{\prime} * f(0)=N_{1}^{\prime \prime} * f(0)=N_{1} * f(0)$, on a $c_{1}=\alpha$ et $c_{2}=1-\alpha$, d'où $N_{1}=N_{1}^{\prime}=N_{1}^{\prime \prime}$. Donc $N_{1} \in \operatorname{Ex}$. $C\left(N^{\prime} ; f\right)$. La démonstration du lemme 8 est ainsi complète.

Corollaire 4. Soit $N_{1}$ un noyau de convolution sur $X N$-invariant et appartenant à $\operatorname{Ex} . C\left(N^{\prime} ; f\right)$; alors on $a$ :

(i) Pour tout $V$ de $\mathscr{V}_{0}$, toute la suite $\left(\omega_{n}\right)_{n=1}^{\infty}$ d'ouverts relativement compacts de $X$ avec $\bar{\omega}_{n} \subset \omega_{n+1}$ et $\bigcup_{n=1}^{\infty} \omega_{n}=X$ et pour un ensemble borélien $B$ de $X$, tout le point vaguement adhérent $\eta$ de $\left(N * \mu_{n, B}^{\prime}\right)_{n=1}^{\infty}$ est proportionnel à $N_{1}$, où $\mu_{n}^{\prime}$ est la mesure balayée de $\varepsilon$ sur $C V \cap \omega_{n}$ relativement à $\left(N, N_{1}\right)$ et $\mu_{n, B}^{\prime}$ est la restreinte de $\mu_{n}^{\prime}$ sur $B$.

(ii) Soient $V,\left(\omega_{n}\right)_{n=1}^{\infty}$ et $B$ les mêmes que ci-dessus; alors tout le point vaguement adhérent $\eta$ de $\left(N_{1} * \varepsilon_{n, B}^{\prime}\right)_{n=1}^{\infty}$ est proportionnel $\grave{a} N_{1}$, où $\varepsilon_{n}^{\prime}$ est la mesure balayée de $\varepsilon$ sur $C V \cap \omega_{n}$ relativement au noyau $N$ et où $\varepsilon_{n, B}^{\prime}$ est la restreinte de $\varepsilon_{n}^{\prime}$ sur $B$.

En effet, soit $\eta$ un point vaguement adhérent de $\left(N * \mu_{n, B}^{\prime}\right)_{n=1}^{\infty}$; alors $\eta$ est un $N$-pseudo-potentiel pur (cf. la proposition 4). On choisit un soussuite $\left(N * \mu_{m, B}^{\prime}\right)_{m=1}^{\infty}$ qui converge vaguement vers $\eta$ dans $X$ avec $n \rightarrow+\infty$. Dans ce cas, on peut supposer que $\left(N * \mu_{m, C B}^{\prime}\right)_{m=1}^{\infty}$ converge vaguement vers une mesure de Radon positive $\eta^{\prime}$ dans $X$ avec $m \rightarrow+\infty$, où $\mu_{m, C B}^{\prime}$ est la restreinte de $\mu_{m}^{\prime}$ sur $C B$. Alors $\eta^{\prime}$ est aussi un $N$-pseudo-potentiel pur, d'après la proposition 4 . Ayant

$$
\lim _{n \rightarrow+\infty} N * \mu_{n}^{\prime}=N_{1} \text { (vaguement), }
$$


on obtient $N_{1}=\eta+\eta^{\prime}$, et donc il existe une constante $c>0$ telle que $\eta=c N_{1}$, d'où l'énoncé (i).

L'énoncé (ii) peut être montré de la même manière que ci-dessus, car $\left(N_{1} * \varepsilon_{n}^{\prime}\right)_{n=1}^{\infty}$ converge d'une manière croissante vers $N_{1}$ avec $n \rightarrow+\infty$.

Lemme 9. On a $N^{\prime} \in \operatorname{Ex} . C\left(N^{\prime} ; f\right)$.

En effet, on a évidemment $N^{\prime} \in C\left(N^{\prime} ; f\right)$. Supposons que $N^{\prime}$ n'est pas extrême; alors il existe deux $N$-pseudo-potentiels purs $N_{1}$ et $N_{2}$ de $C\left(N^{\prime} ; f\right)$ tels que $N_{1} \neq N_{2}$ et $N^{\prime}=\frac{1}{2}\left(N_{1}+N_{2}\right)$. On a $N^{\prime} * f(0)=N_{1} * f(0)$ $=N_{2} * f(0)$, et $N_{1}$ et $N_{2}$ sont de $N^{\prime}$-pseudo-potentiels purs, car d'après le lemme $6, N_{1}$ et $N_{2}$ sont évidemment $N$-invariants, et pour un voisinage $V$ de $\mathscr{V}_{0}$ et pour une suite $\left(\omega_{n}\right)_{n=1}^{\infty}$ d'ouverts relativement compacts avec $\bar{\omega}_{n} \subset \omega_{n+1}$ et $\bigcup_{n=1}^{\infty} \omega_{n}=X$, d'après le corollaire 2 , on a $\varepsilon_{n}^{\prime \prime}=\frac{1}{2}\left(\varepsilon_{1, n}^{\prime}+\varepsilon_{2, n}^{\prime}\right)$, où $\varepsilon_{n}^{\prime \prime}$ et $\varepsilon_{i, n}^{\prime}$ sont respectivement les mesures balayées de sur $C V \cap \omega_{n}$ relativement à $\left(N, N^{\prime}\right)$ et relativement à $\left(N, N_{i}\right)(i=1,2)$, d'où $\left(N_{0} * \varepsilon_{1, n}^{\prime}\right)_{n=1}^{\infty}$ et $\left(N_{0} * \varepsilon_{2, n}^{\prime}\right)_{n=1}^{\infty}$ convergent vaguement vers 0 avec $n \rightarrow+\infty$. Le noyau de convolution $N_{0}+\frac{1}{2}\left(N_{1}+N_{2}\right)$ étant un $N$-pseudo-potentiel pur et $N_{i}$ étant un $N^{\prime}$-pseudo-potentiel pur $(i=1,2)$, il existe deux mesures de Radon positives $\mu_{1}$ et $\mu_{2}$ dans $X$ telles que $N_{0} * \mu_{1}+\frac{1}{2} N_{1}$ et $N_{0} * \mu_{2}+\frac{1}{2} N_{2}$ soient de $N$-pseudo-potentiel purs et que $N_{0}=N_{0} * \mu_{1}+N_{0} * \mu_{2}$. D'après

$$
\frac{N^{\prime} * f(0)}{N * f(0)} N \in \operatorname{Ex} . C\left(N^{\prime} ; f\right) \text {, }
$$

$N_{0} * \mu_{i}+\frac{1}{2} N_{i}$ est proportionnel à $N$, et par suite

$$
N_{0} * \mu_{i}+\frac{1}{2} N_{i}=\frac{2 N_{0} * \mu_{i} * f(0)+N^{\prime} * f(0)}{2 N * f(0)}\left(N_{0}+N^{\prime}\right) \quad(i=1,2) .
$$

D'après l'unicité de la décomposition dans le lemme 6 , on a

$$
\mu_{i}=\frac{2 N_{0} * \mu_{i} * f(0)+N^{\prime} * f(0)}{2 N * f(0)} \varepsilon \quad \text { et } \quad N_{i}=\frac{2 N_{0} * \mu_{i} * f(0)+N^{\prime} * f(0)}{N * f(0)} N^{\prime} .
$$

En utilisant $N^{\prime} * f(0)=N_{1} * f(0)=N_{2} * f(0)$, on a $N^{\prime}=N_{1}=N_{2}$, d'où une contradiction, d'où le lemme 9 .

Montrons que la condition est nécessaire dans notre théorème. On le montrera en séparant en quatre parties suivantes. Soient $N_{0}$ et $N^{\prime}$ aussi la partie régulière de $N$ et la partie singulière de $N$. Si $N_{0}=0$ ou bien $N^{\prime}=0$, alors la condition est évidemment nécessaire, et par suite 
on peut supposer $N_{0} \neq 0$ et $N^{\prime} \neq 0$. Soient $C\left(N^{\prime} ; f\right)$ et $\operatorname{Ex} . C\left(N^{\prime} ; f\right)$ les mêmes que ci-dessus.

(1) Tout le noyau de convolution $N_{1}$ sur $X N$-invariant et appartenant à Ex. $C\left(N^{\prime} ; f\right)$ est pseudo-périodique à tout le point du sous-groupe fermé engendré par supp $\left(N_{0}\right)$.

En effet, supp $\left(N_{0}\right)$ est un semi-groupe fermé dans $X$ et si, pour un point $x$ de $X, N_{1}$ est pseudo-périodique à $x$, alors $N_{1}$ est aussi pseudopériodique au point $-x$. $\mathrm{D}^{\prime}$ autre part, on a

$$
\operatorname{supp}\left(N_{0}\right)=\bigcup_{n=0}^{\infty} \bigcup_{V \in r_{0}} \operatorname{supp}\left(\left(\varepsilon_{C V}^{\prime}\right)^{n}\right),
$$

où $\varepsilon_{C V}^{\prime}$ est la mesure balayée de $\varepsilon$ sur $C V$ relativement au noyau $N_{0}$ et $\left(\varepsilon_{C V}^{\prime}\right)^{0}=\varepsilon,\left(\varepsilon_{C V}^{\prime}\right)^{n}=\left(\varepsilon_{C V}^{\prime}\right)^{n-1} * \varepsilon_{C V}^{\prime}(n \geqq 1)$ (cf. la remarque 2 dans [7]), et la totalité des pseudo-périodes de $N_{1}$ est fermé. Par conséquent, il suffit de montrer que, pour un voisinage quelconque $V$ de $\mathscr{V}_{0}$ et pour un entier $m>0$ quelconque, $N_{1}$ est pseudo-périodique à tout le point de $\operatorname{supp}\left(\left(\varepsilon_{C V}^{\prime}\right)^{m}\right)$. Soit $\left(\omega_{n}\right)_{n=1}^{\infty}$ une suite d'ouverts relativement compacts de $X$ avec $\bar{\omega}_{n} \subset \omega_{n+1}$ et $\bigcup_{n=1}^{\infty} \omega_{n}=X$; on désigne par $\varepsilon_{n}^{\prime}$ la mesure balayée de $\varepsilon \operatorname{sur} C V \cap \omega_{n}$ relativement au noyau $N$. Alors $\left(\varepsilon_{n}^{\prime}\right)_{n=1}^{\infty}$ converge vaguement vers $\varepsilon_{C V}^{\prime}$ avec $n \rightarrow+\infty$ (cf. la proposition 6). D'après le lemme 5 , on a

$$
\lim _{n \rightarrow+\infty} N_{1} *\left(\varepsilon_{n}^{\prime}\right)^{m}=N_{1} \text { (vaguement) . }
$$

Soit $\left(\varepsilon_{C V}^{\prime}\right)_{n}$ la restreinte de $\varepsilon_{C V}^{\prime}$ sur $\omega_{n} ;$ alors $\left(\varepsilon_{n}^{\prime}\right)^{m} \geqq\left(\left(\varepsilon_{C V}^{\prime}\right)_{n}\right)^{m}$ dans $X$. Ayant

$$
N_{1}-N_{1} *\left(\varepsilon_{C V}^{\prime}\right)^{m}=\lim _{n \rightarrow+\infty}\left(N_{1} *\left(\varepsilon_{n}^{\prime}\right)^{m}-N_{1} *\left(\left(\varepsilon_{C V}^{\prime}\right)_{n}\right)^{m}\right)
$$

dans $X$, on obtient que $N_{1}-N_{1} *\left(\varepsilon_{C V}^{\prime}\right)^{m}$ est un $N$-pseudo-potentiel pur. Pour tout $x$ de $\operatorname{supp}\left(\left(\varepsilon_{C V}^{\prime}\right)^{m}\right)$ et pour tout le voisinage ouvert $V(x)$ de $x,\left(\varepsilon_{C V}^{\prime}\right)_{V(x)}^{m}$ désigne la restreinte de $\left(\varepsilon_{C V}^{\prime}\right)^{m}$ sur $V(x)$, et alors cela ne s'annule pas dès que $\varepsilon_{C V}^{\prime} \neq 0$. On peut supposer évidemment $\varepsilon_{C V}^{\prime} \neq 0$ et $N_{1} \neq 0 . \quad N_{1}-N_{1} *\left(\varepsilon_{C V}^{\prime}\right)_{V(x)}^{m}$ est aussi un $N$-pseudo-potentiel pur et on a $N_{1} *\left(\varepsilon_{C V}^{\prime}\right)_{V(x)}^{m} \neq 0$. D'après le lemme 8 , il existe une constante $c_{V(x)}>0$ telle que

$$
c_{V(x)} N_{1} *\left(\varepsilon_{C V}^{\prime}\right)_{V(x)}^{m}=N_{1}
$$

Lorsque $\overline{V(x)}$ tend d'une manière décroissante vers $\{x\}$, la famille 
$\left(c_{V(x)}\left(\varepsilon_{C V}^{\prime}\right)_{V(x)}^{m}\right)$ est vaguement bornée. Done il existe une constante $c_{x}>0$ telle que $c_{x} N_{1} * \varepsilon_{x}=N_{1}$, d'où $N_{1}$ est pseudo-périodique à tout le point de $\operatorname{supp}\left(\left(\varepsilon_{C V}^{\prime}\right)^{n}\right)$.

Comme $N^{\prime} \in \operatorname{Ex} . C\left(N^{\prime} ; f\right)$, d'après le lemme $9, N^{\prime}$ est pseudo-périodique à tout le point de $\operatorname{supp}\left(N_{0}\right)$. Par conséquent, il existe une fonction positive, continue et exponentielle $\psi$ dans $X$ telle que $\psi N^{\prime}$ soit périodique à tout le point du sous-groupe fermé engendré par supp $\left(N_{0}\right)$.

(2) Montrons que $N^{\prime}$ satisfait au principe de domination dans le cas où $\int \psi d N_{0}<+\infty$ et $\operatorname{supp}\left(N_{0}\right) \neq\{0\}$.

D'après le lemme 3 , il suffit de voir que $\psi N^{\prime}$ satisfait au principe de domination. Pour le principe de domination pour $\psi N^{\prime}$, il suffit d'avoir l'implication suivante:

Quelles que soient $g, h$ de $C_{K}^{+}$,

$$
\left(\psi N^{\prime}\right) * g<\left(\psi N^{\prime}\right) * h \quad \text { sur } \quad \operatorname{supp}(g) \Rightarrow\left(\psi N^{\prime}\right) * g \leqq\left(\psi N^{\prime}\right) * h \quad \text { sur } \quad X .
$$

Voir [5]. Posons

$$
a=\min _{x \in \operatorname{supp}(g)}\left(\left(\psi N^{\prime}\right) * h(x)-\left(\psi N^{\prime}\right) * g(x)\right) ;
$$

on choisit ensuite un entier $m>0$ tel que

$$
\frac{1}{m} \max _{x \in \operatorname{supp}(g)}\left(\psi N_{0}\right) * g(x)<a .
$$

D'après $\int \psi d N_{0}<+\infty,\left(\psi N_{0}\right) * g$ s'annule à l'infini, et par suite on peut choisir une famille $\left(x_{i}\right)_{i=1}^{m}$ de points de $\operatorname{supp}\left(N_{0}\right)$ avec $x_{1}=0$ telle que, quels que soient $i, j$ avec $i \neq j$,

$$
\left(\psi N_{0}\right) *\left(g * \varepsilon_{x_{i}}\right)(x)<a-\frac{1}{m} \max _{y \in \text { supp }(g)}\left(\psi N_{0}\right) * g(y)
$$

$\operatorname{sur} \operatorname{supp}\left(g * \varepsilon_{x_{j}}\right)$. Posons

$$
g_{m}=\frac{1}{m} \sum_{i=1}^{m} g * \varepsilon_{x_{i}} ;
$$

on a alors

$$
\max _{x \in \operatorname{supp}\left(f_{m}\right)}\left(\psi N_{0}\right) * g_{m}(x)<a, \quad\left(\psi N^{\prime}\right) * g_{m}=\left(\psi N^{\prime}\right) * g
$$

et 


$$
\min _{x \in \operatorname{supp}\left(f_{m}\right)}\left(\left(\psi N^{\prime}\right) * h(x)-\left(\psi N^{\prime}\right) * g_{m}(x)\right)=a
$$

Done

$$
\left(\psi N_{0}\right) * g_{m}+\left(\psi N^{\prime}\right) * g_{m}<\left(\psi N^{\prime}\right) * h \quad \text { sur } \quad \operatorname{supp}\left(g_{m}\right),
$$

et par suite

$$
\left(\psi N^{\prime}\right) * g \leqq\left(\psi N_{0}\right) * g_{m}+\left(\psi N^{\prime}\right) * g_{m} \leqq\left(\psi N^{\prime}\right) * h \quad \text { sur } \quad X .
$$

Par conséquent, $\psi N^{\prime}$ satisfait au principe de domination, d'où le principe de domination pour $N^{\prime}$.

(3) Montrons que $N^{\prime}$ satisfait au principe de domination dans le cas où $\int \psi d N_{0}=\infty$.

Pour un voisinage $V$ de $\mathscr{V}_{0}$, on désigne par $\varepsilon_{C V}^{\prime}$ la mesure balayée de $\varepsilon$ sur $C V$ relativement au noyau $N_{0}$. Alors $\psi \varepsilon_{C V}^{\prime}$ est la mesure balayée de $\varepsilon$ sur $C V$ relativement au noyau $\psi N_{0}$. Ayant

$$
\psi N^{\prime} \geqq \psi\left(N^{\prime} * \varepsilon_{C V}^{\prime}\right)=\left(\psi N^{\prime}\right) *\left(\psi \varepsilon_{C V}^{\prime}\right),
$$

on a $\int \psi d \varepsilon_{C V}^{\prime} \leqq 1$, et par suite $\psi N_{0}$ est borné. D'après $\int \psi d N_{0}=+\infty$, on a $\int \psi d \varepsilon_{C V}^{\prime}=1$ et $\left(\psi N^{\prime}\right)=\left(\psi N^{\prime}\right) *\left(\psi \varepsilon_{C V}^{\prime}\right)$. On peut supposer que $N_{0}$ est un noyau de convolution de Hunt sur le sous-groupe fermé $X^{\prime}$ engendré par supp $\left(N_{0}\right) . \quad \psi N_{0}$ étant borné sur $X^{\prime}$, on obtient que, quelle que soit $\xi^{\prime}$ une mesure de Haar sur $X^{\prime}, \psi N_{0}+\xi^{\prime}$ satisfait au principe de domination (cf. le lemme 12 dans [7]). D'après $\xi^{\prime}=\xi^{\prime} *\left(\psi \varepsilon_{C V}^{\prime}\right)\left(\forall V \in \mathscr{V}_{0}\right)$, la partie singulière de $\psi N_{0}+\xi^{\prime}$ est égale à $\xi^{\prime}$. Soit $\left(\omega_{n}\right)_{n=1}^{\infty}$ une suite d'ouverts relativement compacts de $X$ avec $\bar{\omega}_{n} \subset \omega_{n+1}$ et $\bigcup_{n=1}^{\infty} \omega_{n}=X$. On désigne par $\varepsilon_{n}^{\prime \prime}$ la mesure balayée de $\varepsilon$ sur $\omega_{n} \cap X^{\prime}$ relativement à $\left(\psi N_{0}+\xi^{\prime}, \xi^{\prime}\right)$, et alors $\xi^{\prime} * \varepsilon_{n}^{\prime \prime}$ converge d'une manière croissante vers $\xi^{\prime}$ dans $X^{\prime}$ avec $n \uparrow+\infty$. Supposons que, pour deux fonctions $f$ et $g$ de $C_{K}^{+}$,

$$
\left(\psi N^{\prime}\right) * f(x) \leqq\left(\psi N^{\prime}\right) * g(x) \quad \text { sur } \quad \operatorname{supp}(f) ;
$$

alors cette inégalité a lieu sur $X^{\prime}+\operatorname{supp}(f)$. On peut supposer que la mesure $\varepsilon_{n}^{\prime \prime}$ obtenue ci-dessus est une mesure de Radon positive dans $X$ d'une manière usuelle. Alors $\left(\psi N^{\prime}\right) * \varepsilon_{n}^{\prime \prime} \leqq \psi N^{\prime}$ dans $X$ et $\left(\left(\psi N^{\prime}\right) * \varepsilon_{n}^{\prime \prime}\right)_{n=1}^{\infty}$ converge d'une manière croissante vers $\psi N^{\prime}$ dans $X$ avec $n \uparrow+\infty$. On a

$$
(\psi N) *\left(\varepsilon_{n}^{\prime \prime} * f\right)(x) \leqq\left(\psi N^{\prime}\right) * f(x) \leqq\left(\psi N^{\prime}\right) * g(x) \quad \text { sur } \quad X^{\prime}+\operatorname{supp}(f)
$$


et $\operatorname{supp}\left(\varepsilon_{n}^{\prime \prime} * f\right) \subset X^{\prime}+\operatorname{supp}(f)$. D'après $\psi N \prec \psi N^{\prime}$, on a

$$
(\psi N) *\left(\varepsilon_{n}^{\prime \prime} * f\right) \leqq\left(\psi N^{\prime}\right) * g \quad \text { sur } \quad X
$$

En faisant $n \rightarrow+\infty$, on arrive à l'inégalité $\left(\psi N^{\prime}\right) * f \leqq\left(\psi N^{\prime}\right) * g$ sur $X$. Par conséquent, $\psi N^{\prime}$ satisfait au principe de domination, d'où le principe de domination pour $N^{\prime}$.

(4) Montrons que $N^{\prime}$ satisfait au principe de domination dans le cas où $\operatorname{supp}\left(N_{0}\right)=\{0\}$.

On peut supposer évidemment $N_{0}=\varepsilon$. Soit $h$ une fonction strictement positive, finie et continue dans $X$ telle que $N^{\prime} * h$ soit fini et continu (cf. le lemme 4). On peut supposer que $h$ est symétrique par rapport à l'origine, et on a $N^{\prime} * h>0$ dans $X$. On choisit une suite $\left(\omega_{n}\right)_{n=1}^{\infty}$ d'ouverts relativement compacts et symétrique par rapport à l'origine dans $X$ avec $\bar{\omega}_{n} \subset \omega_{n+1}$ et $\bigcup_{n=1}^{\infty} \omega_{n}=X$. Pour le principe de domination pour $N^{\prime}$, il suffit de montrer que l'implication suivante a lieu: Quelles que soient $f, g$ de $C_{K}^{+}$,

$$
N^{\prime} * f<N^{\prime} * g \quad \text { sur } \quad \operatorname{supp}(f) \Rightarrow N^{\prime} * f \leqq N^{\prime} * g \quad \text { sur } \quad X .
$$

Posons $A=\left\{x \in X ; N^{\prime} * f(x)<N^{\prime} * g(x)\right\}$. Soit $\mu_{A, n}^{\prime}$ la mesure balayée de $f \xi$ sur $A \cap \omega_{n}$ relativement à $\left(N, N^{\prime}\right)$; alors, d'après $N_{0}=\varepsilon, \mu_{A, n}^{\prime}$ est absolument continue par rapport à $\xi$ et sa densité $f_{A, n}^{\prime}$ appartient à $M_{K}^{+}$. Désignons par $N f_{A, n}^{\prime}$ la densité de $N *\left(f_{A, n}^{\prime} \xi\right)$ par rapport à $\xi$; alors $\left(N f_{A, n}^{\prime}\right)_{n=1}^{\infty}$ est croissante et on a

$$
N^{\prime} * f=\lim _{n \rightarrow+\infty} N f_{A, n}^{\prime}
$$

$\xi$-p.p. dans $A$. Posons $u=\lim _{n \rightarrow+\infty} N f_{A, n}^{\prime}$; alors $u$ (précisément $u \xi$ ) est un $N$-pseudo-potentiel pur. On a, pour tout entier $n>0, f_{A, n}^{\prime} \geqq f_{A, n+1}^{\prime}$ $\xi$-p.p. dans $\omega_{n}$, et donc $\lim _{n \rightarrow+\infty} f_{A, n}^{\prime}$ existe $\xi$-p.p. sur $X$. On note $f_{A}^{\prime}$ sa limite, et alors on peut écrire $u=f_{A}^{\prime}+u^{\prime}$, où $u^{\prime}$ est un $N^{\prime}$-pseudopotentiel pur. Evidemment $u \leqq N^{\prime} * f \xi$-p.p. sur $X$. Si $u=N^{\prime} * f$, alors, d'après $N \prec N^{\prime}$, on a $N^{\prime} * f \leqq N^{\prime} * g$ sur $X$ (cf. le théorème 1 dans [5]), et donc on montrera $u=N^{\prime} * f \xi$-p.p. sur $X$. Montrons d'abord que $u$ est un $N^{\prime}$-pseudo-potentiel pur. Pour un point $x$ de $A$, on note respectivement $\varepsilon_{x, n}^{\prime \prime}$ et $\left(\varepsilon_{x, n}^{\prime \prime}\right)_{A}$ la mesure balayée de $\varepsilon_{x}$ sur $\omega_{n}$ relativement à $\left(\check{N}, \check{N}^{\prime}\right)$ et la restreinte de $\varepsilon_{x, n}^{\prime \prime}$ sur $A$. On a alors, pour tout $x$ de $A$, 


$$
\begin{array}{rl}
N^{\prime} * & f(x)-N^{\prime} * g(x)=\left(\check{N}^{\prime} * \varepsilon_{x}\right) *(\check{f}-\check{g})(0) \\
& =\lim _{n \rightarrow+\infty}\left(\check{N}^{\prime} * \varepsilon_{x, n}^{\prime \prime}\right) *(\check{f}-\check{g})(0)=\lim _{n \rightarrow+\infty} \int\left(N^{\prime} * f(y)-N^{\prime} * g(y)\right) d \varepsilon_{x, n}^{\prime \prime}(y) \\
& \geqq \varlimsup_{n \rightarrow+\infty} \int\left(N^{\prime} * f(y)-N^{\prime} * g(y)\right) d\left(\varepsilon_{x, n}^{\prime \prime}\right)_{A}(y)=\varlimsup_{n \rightarrow+\infty}\left(\check{N}^{\prime} *\left(\varepsilon_{x, n}^{\prime \prime}\right)_{A}\right) *(\check{f}-\check{g})(0) .
\end{array}
$$

D'après le corollaire 4 , tout le point vaguement adhérent de $\left(\check{N}^{\prime} *\left(\varepsilon_{x, n}^{\prime \prime}\right)_{A}\right)_{n=1}^{\infty}$ est $\leqq \check{N}^{\prime} * \varepsilon_{x}$ et proportionnel à $\check{N}^{\prime} * \varepsilon_{x}$. Donc il existe une constante $c$ avec $0 \leqq c \leqq 1$ telle que

$$
\begin{aligned}
& \varliminf_{n \rightarrow+\infty}\left(\check{N}^{\prime} *\left(\varepsilon_{x, n}^{\prime \prime}\right)_{A}\right) *(\check{f}-\check{g})(0)=c\left(\check{N}^{\prime} * \varepsilon_{x}\right) *(\check{f}-\check{g})(0) \\
& \quad=c\left(N^{\prime} * f(x)-N^{\prime} * g(x)\right) \geqq N^{\prime} * f(x)-N^{\prime} * g(x),
\end{aligned}
$$

d'où $c=1$. Il en résulte que $\left(N^{\prime} *\left(\varepsilon_{x, n}^{\prime \prime}\right)_{A}\right)_{n=1}^{\infty}$ converge vaguement vers $\check{N}^{\prime} * \varepsilon_{x}$ avec $n \rightarrow+\infty$. Pour une fonction $s$ de $M_{K}^{+}$portée par $A$, on désigne par $s_{n}^{\prime \prime}$ et par $\left(s_{n}^{\prime \prime}\right)_{A}$ la densité de la mesure balayée de $s \xi$ sur $\omega_{n}$ relativement à $\left(\check{N}, \check{N}^{\prime}\right)$ et sa restreinte sur $A$, respectivement. Alors $s_{n}^{\prime \prime} \in M_{K}^{+}$et on a

$$
\begin{gathered}
\int\left(N^{\prime} * f(x)-u^{\prime}(x)\right) s(x) d \xi(x)=\lim _{n \rightarrow+\infty} \int\left(N^{\prime} * f(x)-u(x)\right) s_{n}^{\prime \prime}(x) d \xi(x) \\
=\lim _{n \rightarrow+\infty} \int\left(N^{\prime} * f(x)-u(x)\right)\left(s_{n}^{\prime \prime}\right)_{A}(x) d \xi(x)=0
\end{gathered}
$$

car

$$
\left(s_{n}^{\prime \prime}\right)_{A} \xi=\int \varepsilon_{x, A}^{\prime \prime} s(x) d \xi(x)
$$

Par conséquent, quelle que soit $s$ une fonction de $M_{K}^{+}$portée par $A$,

$$
\int f_{A}^{\prime}(x) s(x) d \xi(x)=0
$$

d'où, d'après $\operatorname{supp}\left(f_{A}^{\prime}\right) \subset \bar{A}$, on a $f_{A}^{\prime}=0$. On obtient ainsi que $u$ est un $N^{\prime}$-pseudo-potentiel pur.

Montrons ensuite que, quelle que soit $s$ de $M_{K}$,

$$
\int N^{\prime}\left(\frac{s}{N^{\prime} * h}\right)(x) \frac{s(x)}{N^{\prime} * h(x)} d \xi(x) \geqq 0 \text {. }
$$

Soit $\mathscr{E}$ la $\sigma$-algèbre constituée par tous les ensembles $\xi$-mesurables de $X$; on pose, pour tous $e_{1}, e_{2}$ de $\mathscr{E}$, 


$$
G\left(e_{1}, e_{2}\right)=\int N\left(\frac{c_{2}}{N^{\prime} * h}\right)(x) \frac{c_{1}(x)}{N^{\prime} * h(x)} d \xi(x)
$$

et

$$
G^{\prime}\left(e_{1}, e_{2}\right)=\int N^{\prime}\left(\frac{c_{2}}{\check{N}^{\prime} * h}\right)(x) \frac{c_{1}(x)}{N^{\prime} * h(x)} d \xi(x)
$$

où $c_{i}(i=1,2)$ est la fonction caractéristique de $e_{i}$. Alors $G$ et $G^{\prime}$ sont de noyaux relatifs à $X$ et à $\xi$ (voir [4]). On note $\breve{G}\left(e_{2}, e_{1}\right)=G\left(e_{2}, e_{1}\right)$ et $\check{G}^{\prime}\left(e_{1}, e_{2}\right)=G^{\prime}\left(e_{1}, e_{2}\right) \quad\left(\forall e_{1}, \forall e_{2} \in \mathscr{E}\right)$. Pour une fonction $s$ de $M_{K}^{+}$, le potentiel $G s$ de $s$ par rapport au noyau $G$ et le potentiel $\breve{G} s$ de $s$ par rapport au noyau $\breve{G}$ sont de la forme

$$
G s=\frac{1}{N^{\prime} * h} N\left(\frac{s}{N^{\prime} * h}\right) \text { et } \quad G s=\frac{1}{\check{N}^{\prime} * h} \check{N}\left(\frac{s}{N^{\prime} * h}\right),
$$

et donc, d'après le principe de domination pour $N$ et $\check{N}, N \prec N^{\prime}$ et $\check{N} \prec$ $\check{N}^{\prime}, G$ et $\check{G}$ satisfont au principe complet $d u$ maximum ${ }^{2}$. Par conséquent, $G$ est de type positif, c'est-à-dire, pour toute $s$ de $M_{K}$,

$$
\int G s(x) s(x) d \xi(x) \geqq 0 \text {. }
$$

Cela est un résultat principal de l'article [4]. De la même manière que dans le cas où $G$ est un noyau de convolution borné (cf. la démonstration du théorème dans [7]), on obtient que $G^{\prime}$ est aussi de type positif, d'où, pour toute $s$ de $M_{K}$,

$$
\int N^{\prime}\left(\frac{s}{\check{N}^{\prime} * h}\right)(x) \frac{s(x)}{N^{\prime} * h(x)} d \xi(x) \geqq 0 .
$$

On montrera finalement $N^{\prime} * f=u \xi$-presque partout sur $X$. On a

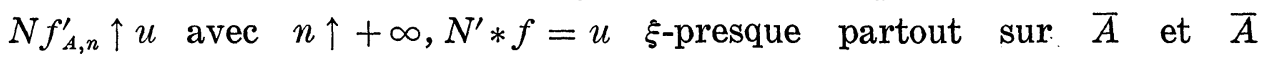
$\supset \operatorname{supp}\left(f_{A, n}^{\prime}\right) \cup \operatorname{supp}(f)(n=1,2, \cdots)$. Donc, en utilisant le fait que $G^{\prime}$ est de type positif, on a

$$
\begin{aligned}
0 & =\lim _{n \rightarrow+\infty} \int G^{\prime}\left(\left(\check{N}^{\prime} * h\right)\left(f-f_{A, n}^{\prime}\right)\right)(x) \check{N}^{\prime} * h(x)\left(f(x)-f_{A, n}^{\prime}(x)\right) d \xi(x) \\
& =\lim _{n \rightarrow+\infty} \int \frac{1}{2}\left(G^{\prime}+\check{G}^{\prime}\right)\left(\left(\check{N}^{\prime} * h\right)\left(f-{f_{A, n}^{\prime}}^{\prime}\right)(x) \check{N}^{\prime} * h(x)\left(f(x)-f_{A, n}^{\prime}(x)\right) d \xi(x) .\right.
\end{aligned}
$$

2) $G$ satisfait au principe complet du maximum si, quelles que soient $f, g$ de $M_{K}^{+}$, l'inégalité $G f \leqq G g+1$ est satisfait $\xi$-p.p. sur $X$ dès qu'elle l'est $\xi$-p.p. sur $\{x \in X$; $f(x)>0\}$. 
D'après le fait que $\frac{1}{2}\left(G^{\prime}+\check{G}^{\prime}\right)$ est un noyau symétrique et de type positif, on a

$$
\lim _{n \rightarrow+\infty}\left(G^{\prime}+\check{G}^{\prime}\right)\left(\left(\check{N}^{\prime} * h\right)\left(f-f_{A, n}^{\prime}\right)\right)(x)=0
$$

$\xi$-p.p. sur $X$, et par suite

$$
\frac{N^{\prime} * h}{\check{N}^{\prime} * h}\left(\check{N^{\prime}} *\left(\frac{\check{N^{\prime}} * h}{N^{\prime} * h} f\right)\right)-\frac{N^{\prime} * h}{\check{N^{\prime} * h}} \lim _{n \rightarrow+\infty} \check{N^{\prime}}\left(\frac{\check{N^{\prime}} * h}{N^{\prime} * h} f_{A, n}^{\prime}\right)=u-N^{\prime} * f
$$

$\xi$-p.p. $\operatorname{sur} X$. La suite $\left(f_{A, n}^{\prime}\right)$ convergeant vers $0 \xi$-p.p. sur $X$ et ayant

$$
\lim _{n \rightarrow+\infty} \check{N}^{\prime}\left(\frac{\check{N^{\prime} * h}}{N^{\prime} * h} f_{A, n}^{\prime}\right)=\check{N^{\prime}} *\left(\frac{\check{N}^{\prime} * h}{N^{\prime} * h} f\right)
$$

$\xi$-p.p. $\operatorname{sur} \bar{A} \supset \operatorname{supp}\left(f_{A, n}\right)(n=1,2, \cdots)$, on a, pour tout entier $m \geqq 1$,

$$
\lim _{n \rightarrow+\infty}\left(\check{N}^{\prime}\left(\frac{\check{N}^{\prime} * h}{N^{\prime} * h} f_{A, n}^{\prime}\right)-\check{N}^{\prime}\left(\frac{\check{N}^{\prime} * h}{N^{\prime} * h} f_{A, n, m}^{\prime}\right)\right)=0
$$

$\xi-$ p.p. $\operatorname{sur} X$, où $f_{A, n, m}^{\prime}$ est la restreinte de $f_{A, n}^{\prime}$ sur

$$
\left\{x \in X ; \check{N}\left(\frac{\check{N^{\prime}} * h}{N^{\prime} * h} f_{A, n}^{\prime}\right)(x) \leqq \check{N^{\prime}} *\left(\frac{\check{N}^{\prime} * h}{N^{\prime} * h} f\right)(x)+\frac{1}{m} \check{N}^{\prime} * h(x)\right\}
$$

D'après $\check{N} \prec \check{N}^{\prime}$, on a

$$
\check{N}^{\prime}\left(\frac{\check{N}^{\prime} * h}{N^{\prime} * h} f_{A, n, m}^{\prime}\right) \leqq \check{N}\left(\frac{\check{N} \check{N}^{\prime} * h}{N^{\prime} * h} f_{A, n, m}^{\prime}\right) \leqq \check{N^{\prime}} *\left(\frac{\check{N}^{\prime} * h}{N^{\prime} * h} f\right)+\frac{1}{m} \check{N}^{\prime} * h
$$

$\xi$-p.p. $\operatorname{sur} X$ (cf. le théorème 1 dans [5]). En faisant $n \uparrow+\infty$ et ensuite $m \uparrow+\infty$, on arrive à l'inégalité

$$
\lim _{n \rightarrow+\infty} \check{N^{\prime}}\left(\frac{\check{N}^{\prime} * h}{N^{\prime} * h} f_{A, n}^{\prime}\right) \leqq \check{N^{\prime}} *\left(\frac{\check{N}^{\prime} * h}{N^{\prime} * h} f\right)
$$

$\xi$-p.p. sur $X$, et par suite $u \geqq N^{\prime} * f$, d'où $u=N^{\prime} * f \xi$-p.p. sur $X$. On obtient ainsi que $N^{\prime}$ satisfait au principe de domination dans le cas où $\operatorname{supp}\left(N_{0}\right)=\{0\}$.

En posant $\varphi=1 / \psi, \widetilde{N}_{0}=\psi N_{0}$ et $\widetilde{N}^{\prime}=\psi N^{\prime}$, on obtient que la condition est nécessaire dans notre théorème.

On montrera que, dans notre théorème, la condition est suffisante. On préparera d'abord le lemme suivant:

LEMME 10. Soient $N_{0}$ un noyau de convolution borné de Hunt sur $X$ et $N^{\prime}$ un noyau de convolution $(\neq 0)$ sur $X$ satisfaisant au principe de 
domination et périodique à tout le point de $\operatorname{supp}\left(N_{0}\right)$. Alors, pour une mesure de Radon positive $\mu$ dans $X$ et pour un ouvert relativement compact $\omega$ de $X$, il existe une mesure de Radon positive $\mu^{\prime}$ dans $X$ portée par $\bar{\omega}$ telle que, au sens des mesures, $\left(N_{0}+N^{\prime}\right) * \mu^{\prime} \leqq N^{\prime} * \mu$ dans $X$ et $\left(N_{0}+N^{\prime}\right) * \mu^{\prime}=N^{\prime} * \mu$ dans $\omega$.

En effet, on note $N=N_{0}+N^{\prime}$. D'après le théorème 6 dans [8], on a $N_{0} \prec N$, car $N^{\prime}$ est $N_{0}$-harmonique ou un $N_{0}$-potentiel d'une mesure de Radon positive d'accord avec $\int d N_{0}=+\infty$ ou bien $\int d N_{0}<+\infty$. On a aussi $\check{N}_{0} \prec \check{N}$. On a ensuite $N \prec N^{\prime}$. En effet, si, pour deux fonctions $f$ et $g$ de $C_{K}^{+}, N * f<N^{\prime} * g$ sur $\operatorname{supp}(f)$, alors $N^{\prime} * f<N^{\prime} * g \operatorname{sur} \operatorname{supp}(f)$, et par suite $N^{\prime} * f \leqq N^{\prime} * g$ partout sur $X$, d'après le principe de domination pour $N^{\prime}$. Donc $N^{\prime} * g-N^{\prime} * f$ est non-négative et $N_{0}$-harmonique ou un $N_{0}$-potentiel d'une mesure de Radon positive dans $X$ d'accord avec $\int d N_{0}=+\infty$ ou bien $\int d N_{0}<+\infty$.

Soient $h$ une fonction strictement positive, finie et continue dans $X$ symétrique par rapport à l'origine telle que $\left(N^{\prime}+\check{N}^{\prime}\right) * h$ soit fini et continu, et $\mathscr{E}$ la même que ci-dessus. Pour les noyaux de convolution $N_{0}$ et $N^{\prime}$ sur $X$, on définit, de la même manière que ci-dessus, deux noyaux $G_{0}$ et $G^{\prime}$ relatifs à $X$ et à $\xi$; par exemple,

$$
G_{0}\left(e_{1}, e_{2}\right)=\int N_{0}\left(\frac{c_{2}}{\check{N}^{\prime} * h}\right)(x) \frac{c_{1}(x)}{N^{\prime} * h(x)} d \xi(x) \quad\left(e_{1}, e_{2} \in \mathscr{E}\right)
$$

où $c_{i}$ est la fonction caractéristique de $e_{i}(i=1,2)$. $D$ 'après $N_{0} \prec N$ et $\check{N}_{0} \prec \check{N}, G_{0}$ et $\check{G}_{0}$ satisfont au principe complet du maximum ${ }^{11}$. D'après le principe de domination pour $N^{\prime}$ et $\check{N}^{\prime}, G^{\prime}$ et $\check{G}^{\prime}$ satisfont aussi au principe complet du maximum. Posons $G=G_{0}+G^{\prime}$; alors $G$ est de type positif. De la même manière que dans la proposition 2 dans [7], on obtient que, pour une constante $c>0$, une compact $K$ de $X$ et pour une fonction réelle, $\xi$-mesurable et localement bornée $u$ dans $X$, il existe une fonction $f_{c, K, u}$ de $M_{K}^{+}$portée par $K$, et une seule telle que

$$
\begin{gathered}
G f_{c, K, u}+c f_{c, K, u} \geqq u \xi \text {-p.p. } \quad \text { sur } K, \\
G f_{c, K, u}+c f_{c, K, u}=u \xi \text {-p.p. } \quad \text { sur } \quad\left\{x \in X ; f_{c, K, u}(x)>0\right\} .
\end{gathered}
$$

Soit $\left(\varphi_{\alpha}\right)_{\alpha \in \Lambda}$ une famille filtrante de fonctions de $C_{K}^{+}$portées par un compact fixé telle que $\left(\varphi_{\alpha} \xi\right)_{\alpha \in A}$ converge vaguement vers $\varepsilon$. On désigne par $f_{n, \alpha}^{\prime}$ la fonction obtenue ci-dessus pour $c=1 / n, K=\bar{\omega}$ et pour $u$ 
$=G^{\prime}\left(\left(N^{\prime} * h\right) \mu * \varphi_{\alpha}\right)$. Alors, d'après $N \prec N^{\prime}$, on a

$$
N\left(\left(N^{\prime} * h\right) f_{n, \alpha}^{\prime}\right)(x)+\frac{1}{n} N^{\prime} * h(x) f_{n, \alpha}^{\prime}(x) \leqq N^{\prime} *\left(\mu * \varphi_{\alpha}\right)(x)
$$

$\xi-$ p.p. sur $X$ et

$$
N\left(\left(N^{\prime} * h\right) f_{n, \alpha}^{\prime}\right)(x)+\frac{1}{n} N^{\prime} * h(x) f_{n, \alpha}^{\prime}(x)=N^{\prime} *\left(\mu * \varphi_{\alpha}\right)(x)
$$

$\xi$-presque partout sur $\bar{\omega}$. Pour tout $n>0$, la famille $\left(f_{n, \alpha}^{\prime}\right)_{\alpha \in A}$ est évidemment vaguement bornée, et on choisit un point vaguement adhérent $\mu_{n}^{\prime}$ de $\left(f_{n, \alpha}^{\prime} \xi\right)_{\alpha \in \Lambda}$. $\quad N$ étant non-zéro, $\left(\mu_{n}^{\prime}\right)_{n=1}^{\infty}$ est vaguement bornée. Soit $\mu^{\prime}$ un point vaguement adhérent de $\left(\mu_{n}^{\prime}\right)$; alors on a, au sens des mesures,

$$
N * \mu^{\prime} \leqq N^{\prime} * \mu \text { dans } X \text { et } N * \mu^{\prime}=N^{\prime} * \mu \text { dans } \omega
$$

Montrons que la condition est suffisante dans notre théorème. Il suffit de montrer le principe de domination pour $N$ dans le cas où $N$ $=\varphi\left(\widetilde{N_{0}}+\widetilde{N^{\prime}}\right), \widetilde{N_{0}} \neq 0$ et $\widetilde{N^{\prime}} \neq 0$. D'après le lemme 3 , il suffit que $\widetilde{N_{0}}+\widetilde{N^{\prime}}$ satisfasse au principe de domination. Supposons que, pour deux fonctions $f$ et $g$ de $C_{K}^{+}$,

$$
\left(\widetilde{N}_{0}+\widetilde{N}^{\prime}\right) * f(x) \leqq\left(\widetilde{N}_{0}+\widetilde{N^{\prime}}\right) * g(x) \quad \operatorname{sur} \quad \operatorname{supp}(f) .
$$

Soit $x$ un point quelconque de $X$. D'après le lemme 10, il existe un mesure de Radon positive $\varepsilon_{x}^{\prime}$ portée par $\operatorname{supp}(f)$ telle que, au sens des mesures, $(\check{\widetilde{N}}+\check{\widetilde{N}}) * \varepsilon_{x}^{\prime} \leqq \check{\widetilde{N^{\prime}}} * \varepsilon_{x}$ et $\left(\check{\widetilde{N}}_{0}+\check{\widetilde{N^{\prime}}}\right) * \varepsilon_{x}^{\prime}=\check{\widetilde{N}}^{\prime} * \varepsilon_{x}$ dans $\{x \in X ; f(x)$ $>0\}$. On a alors

$$
\begin{aligned}
\widetilde{N}^{\prime} * g(x) & =\int g(y) d\left(\check{\widetilde{N}}^{\prime} * \varepsilon_{x}\right)(y) \geqq \int g(y) d\left(\left(\check{\widetilde{N}}_{0}+\check{\widetilde{N}}^{\prime}\right) * \varepsilon_{x}^{\prime}\right)(y) \\
& =\int\left(\widetilde{N_{0}}+\widetilde{N}^{\prime}\right) * g(y) d \varepsilon_{x}^{\prime}(y) \geqq \int\left(\widetilde{N}_{0}+\widetilde{N}^{\prime}\right) * f(y) d \varepsilon_{x}^{\prime}(y) \\
& =\int f(y) d\left(\left(\check{\widetilde{N}}_{0}+\widetilde{\widetilde{N}}^{\prime}\right) * \varepsilon_{x}^{\prime}\right)(y)=\int f(y) d\left(\widetilde{\widetilde{N}}^{\prime} * \varepsilon_{x}\right)(y)=N^{\prime} * f(x),
\end{aligned}
$$

d'où $\widetilde{N^{\prime}} * g \leqq \widetilde{N^{\prime}} * f$ dans $X$. De la même manière que dans la démonstration de $N \prec N^{\prime}$ dans le lemme 10, on a

$$
\left(\widetilde{N}_{0}+\widetilde{N}^{\prime}\right) * f(x) \leqq\left(\widetilde{N}_{0}+\widetilde{N}^{\prime}\right) * g(x) \text { sur } \quad X,
$$

d'où le principe de domination pour $N$. 
On discutera finalement sur l'unicité la décomposition de $N$. Supposons que $N$ est des deux formes

$$
N=\varphi\left(\widetilde{N}_{0}+\widetilde{N^{\prime}}\right) \quad \text { et } \quad N=\varphi^{\prime}\left(\widetilde{\widetilde{N}}_{0}+\widetilde{\widetilde{N}}^{\prime}\right)
$$

avec $\widetilde{N}_{0} \neq 0$ et $\widetilde{N_{0}} \neq 0$, qui vérifient les conditions dans le théorème. On peut supposer ici que $\varphi \widetilde{N}_{0}$ et $\varphi \widetilde{N}^{\prime}$ sont respectivement la partie régulière et la partie singulière de $N . \operatorname{Si} \operatorname{supp}\left(\widetilde{N}_{0}\right)=\{0\}$, alors, d'après la singularité de $\widetilde{N^{\prime}}$ et le lemme 3 , supp $\left(\widetilde{\widetilde{N}}_{0}\right)=\{0\}$, et on obtient immédiate ment $\widetilde{N}_{0}=\widetilde{\widetilde{N}}_{0}$ et $\varphi \widetilde{N}^{\prime}=\varphi^{\prime} \widetilde{\widetilde{N}^{\prime}}$. Donc on peut supposer $\operatorname{supp}\left(N_{0}\right) \neq\{0\}$. On a évidemment $\widetilde{\widetilde{N}_{0}} \neq 0, \widetilde{\widetilde{N}^{\prime}} \neq 0$ et $N \prec \varphi^{\prime} \widetilde{\widetilde{N}}^{\prime}$ (cf. le lemme 3), et donc, d'après le lemme 6 , on peut écrire uniquement $\widetilde{N^{\prime}}=\left(\frac{\varphi}{\varphi^{\prime}}\left(\widetilde{N_{0}}+\widetilde{N^{\prime}}\right)\right) * \mu$ $+\eta$, où $\mu$ est une mesure de Radon positive dans $X$ et $\eta$ est un $\left(\frac{\varphi}{\varphi^{\prime}}\left(\widetilde{N}_{0}+\widetilde{N^{\prime}}\right)\right)$-pseudo-potentiel pur et $\left(\frac{\varphi}{\varphi^{\prime}}\left(\widetilde{N}_{0}+\widetilde{N^{\prime}}\right)\right)$-invariant. Pour un voisinage $V$ de $\mathscr{V}_{0}$, on désigne par $\varepsilon_{C V}^{\prime \prime}$ la mesure balayée de $\varepsilon$ sur $C V$ relativement au noyau $\widetilde{\widetilde{N}}_{0}$. Alors $\left(\frac{\varphi}{\varphi^{\prime}} \widetilde{N}_{0}\right) * \varepsilon_{C V}^{\prime \prime}$ et $\left(\frac{\varphi}{\varphi^{\prime}} \widetilde{N}^{\prime}\right) * \varepsilon_{C V}^{\prime \prime}$ sont définies et on a

$$
\begin{aligned}
\widetilde{\widetilde{N}}_{0} *\left(\varepsilon-\varepsilon_{C V}^{\prime \prime}\right)= & \left(\frac{\varphi}{\varphi^{\prime}} \widetilde{N_{0}}\right) *\left(\varepsilon-\varepsilon_{C V}^{\prime \prime}\right)+\left(\frac{\varphi}{\varphi^{\prime}} \widetilde{N^{\prime}}\right) *\left(\varepsilon-\varepsilon_{C V}^{\prime \prime}\right)-\widetilde{\widetilde{N}^{\prime}} *\left(\varepsilon-\varepsilon_{C V}^{\prime \prime}\right) \\
= & \left(\frac{\varphi}{\varphi^{\prime}} \widetilde{N_{0}}\right) *(\varepsilon-\mu) *\left(\varepsilon-\varepsilon_{C V}^{\prime \prime}\right)+\left(\frac{\varphi}{\varphi^{\prime}} \widetilde{N^{\prime}}\right) *(\varepsilon-\mu) *\left(\varepsilon-\varepsilon_{C V}^{\prime \prime}\right) \\
& -\eta *\left(\varepsilon-\varepsilon_{C V}^{\prime \prime}\right) .
\end{aligned}
$$

La partie régulière de $\frac{\varphi}{\varphi^{\prime}} N$ étant égale à $\frac{\varphi}{\varphi^{\prime}} N_{0}$ (cf. le lemme 3) et $\operatorname{supp}\left(\widetilde{\tilde{N}_{0}}-\widetilde{N}_{0} * \varepsilon_{C V}^{\prime \prime}\right)$ étant compact, on a

$$
\widetilde{\widetilde{N}}_{0} *\left(\varepsilon-\varepsilon_{C V}^{\prime \prime}\right)=\left(\frac{\varphi}{\varphi^{\prime}} \widetilde{N}_{0}\right) *(\varepsilon-\mu) *\left(\varepsilon-\varepsilon_{C V}^{\prime \prime}\right)
$$

et

$$
\left(\frac{\varphi}{\varphi^{\prime}} \widetilde{N^{\prime}}\right) *(\varepsilon-\mu) *\left(\varepsilon-\varepsilon_{C V}^{\prime \prime}\right)-\eta *\left(\varepsilon-\varepsilon_{C V}^{\prime \prime}\right)=0
$$

On obtient ensuite que, pour toute $f$ de $C_{K}^{+},\left(\frac{\varphi}{\varphi^{\prime}} \widetilde{N_{0}}\right) *(\varepsilon-\mu) * f$ et 
$\left(\frac{\varphi}{\varphi^{\prime}} \widetilde{N^{\prime}}\right) *(\varepsilon-\mu) * f$ sont bornées sur le sous-groupe fermé engendré par $\operatorname{supp}\left(\widetilde{\widetilde{N}_{0}}\right)$.

Supposons d'abord $\int d \widetilde{\widetilde{N}}_{0}<+\infty$. Alors $\int d \varepsilon_{C V}^{\prime \prime}<1$, et par suite

$$
\widetilde{\widetilde{N}}_{0}=\left(\frac{\varphi}{\varphi^{\prime}} \tilde{N}_{0}\right) *(\varepsilon-\mu)
$$

d'où

$$
\frac{\varphi}{\varphi^{\prime}} \tilde{N}_{0}=\tilde{N}_{0}+\tilde{N}_{0} *\left(\sum_{n=1}^{\infty}(\mu)^{n}\right) .
$$

Par conséquent, $\operatorname{supp}\left(\tilde{\widetilde{N}}_{0}\right) \subset \operatorname{supp}\left(\tilde{N}_{0}\right)$. D'après le fait que, pour toute $f$ de $C_{K}^{+},\left(\frac{\varphi}{\varphi^{\prime}} \tilde{N}^{\prime}\right) * f$ est bornée sur le sous-groupe fermé engenré par $\operatorname{supp}\left(\widetilde{\widetilde{N}}_{0}\right)$ et le fait que $\left(\frac{\varphi}{\varphi^{\prime}} \tilde{N}^{\prime}\right)$ est pseudo-périodique à tout le point de $\operatorname{supp}\left(\tilde{N}_{0}\right),\left(\frac{\varphi}{\varphi^{\prime}} \tilde{N}^{\prime}\right) * \mu+\eta$ est périodique à tout le point de $\operatorname{supp}\left(\widetilde{\tilde{N}}_{0}\right)$. Par conséquent, $\left(\frac{\varphi}{\varphi^{\prime}} \tilde{N}_{0}\right) * \mu$ est périodique à tout le point de $\operatorname{supp}\left(\tilde{\tilde{N}}_{0}\right)$, et par suite $\widetilde{N}_{0} * \sum_{n=1}^{\infty}(\mu)^{n}$ l'est aussi, d'où $\sum_{n=1}^{\infty}(\mu)^{n}$ l'est aussi. Le noyau de convolution $\varepsilon+\sum_{n=1}^{\infty}(\mu)^{n}$ étant un noyau de convolution de Hunt sur $X$, on a $\mu=0$, d'où $\varphi \tilde{N}_{0}=\varphi^{\prime} \tilde{N}_{0}$ et $\varphi \tilde{N}^{\prime}=\varphi^{\prime} \tilde{N}^{\prime}$.

Supposons ensuite $\int d \widetilde{N}_{0}=+\infty$; alors $\int d \varepsilon_{C V}^{\prime \prime}=1$. D'après l'égalité $\tilde{N}^{\prime}=\tilde{\tilde{N}^{\prime}} * \varepsilon_{\sigma V}^{\prime \prime}$, on a

$$
\widetilde{N}_{0} *\left(\varepsilon-\varepsilon_{C V}^{\prime \prime}\right)=\left(\frac{\varphi}{\varphi^{\prime}} \tilde{N}_{0}\right) *\left(\varepsilon-\varepsilon_{C V}^{\prime \prime}\right),
$$

et par suite $\widetilde{\widetilde{N}}_{0}=\varphi / \varphi^{\prime} \tilde{N}_{0}$, d'où $\varphi \tilde{N}_{0}=\varphi^{\prime} \widetilde{N}_{0}$ et $\varphi \tilde{N}^{\prime}=\varphi^{\prime} \tilde{N}^{\prime}$.

Ayant $\tilde{N}^{\prime}=\varphi^{\prime} / \varphi \tilde{N^{\prime}}$, on obtient facilement que $\varphi=\varphi^{\prime}$ sur le sousgroupe fermé engendré par $\operatorname{supp}\left(\varphi \tilde{N}_{0}\right)$.

La démonstration de notre théorème est ainsi complète.

Remarque 5. Soit $N_{0}$ un noyau de convolution de Hunt sur $X$. S'il existe un noyau de convolution $N$ sur $X$ satisfaisant au principe de domination dont la partie régulière est égale à $N_{0}$ et dont la partie singulière ne s'annule pas, alors il existe une fonction positive, continue 
et exponentielle $\varphi$ dans $X$ telle que $\varphi N_{0}$ soit borné, d'après le présent théorème.

\section{Appendice}

Le but de ce paragraphe est de discuter notre problème sans notre hypothèse qu'il n'existe aucun sous-groupe compact de $X$ excepté $\{0\}$. Dans cette section, on ne suppose aucune condition additionnelle pour $X$. Dans ce cas, le présent théorème est de la forme suivante:

Proposition 9. Soit $N$ un noyau de convolution $(\neq 0)$ sur $X$. Alors pour que $N$ satisfasse au principe de domination, il faut et il suffit qu'il existe un sous-groupe compact $\Gamma$ de $X$ telle que $N$ soit le prolongement canonique de $\bar{\varphi}\left(\bar{N}_{0}+\bar{N}^{\prime}\right)$ sur $X$, où $\bar{\varphi}$ est une fonction positive, continue et exponentielle dans $X / \Gamma, \bar{N}_{0}$ est un noyau de convolution de Hunt sur $X / \Gamma$ ou bien $\bar{N}_{0}=0$ et où $\bar{N}^{\prime}$ est un noyau de convolution singulier sur $X / \Gamma$ (satisfaisant au principe de domination) avec $\bar{N}_{0} \prec \bar{N}^{\prime}$ et périodique à tout le point de $\operatorname{supp}\left(\bar{N}_{0}\right)$.

Démonstration. Voyons d'abord que la condition est suffisante. D'après le présent théorème, le noyau de convolution $\bar{\varphi}\left(\bar{N}_{0}+\bar{N}^{\prime}\right)$ sur $X / \Gamma$ satisfait au principe de domination. On remarque ici que, d'après $\bar{N}_{0}$ $\prec \bar{N}^{\prime}, \bar{N}_{0}$ est borné dès que $\bar{N}^{\prime} \neq 0$. Donc on obtient facilement le principe de domination pour $N$. Par conséquent, on montrera seulement que la condition est nécessaire.

On pose, pour un entier $n \geqq 1, N_{n}=\frac{1}{n} \varepsilon+N$. Alors $N_{n}$ satisfait au principe de domination. De la même manière que ci-dessus, on peut définir la partie régulière $N_{0, n}$ de $N_{n}$ et la partie singulière $N_{n}^{\prime}$ de $N_{n}$. Pour un voisinage $V$ de $\mathscr{V}_{0}$, on note $\eta_{n, C V}$ et $\eta_{C V}$ le $N_{n}$-pseudo-potentiel balayé de $N_{n}$ sur $C V$ relativement au noyau $N_{n}$ et le $N$-pseudo-potentiel balayé de $N$ sur $C V$ relativement au noyau $N$, respectivement. Si $n \geqq m$, alors $N_{m} \prec N_{n} \prec N$, et par suite la suite $\left(\eta_{n, C V}\right)_{n=1}^{\infty}$ est croissante. Donc $\left(N_{0, n}\right)_{n=1}^{\infty}$ est décroissante et on a $\lim _{n \uparrow+\infty} N_{0, n} \geqq N_{0}$, où $N_{0}$ est la partie régulière de $N$. D'après le principe de domination pour $N_{0, n}\left({ }^{\forall} \geqq 1\right)$, $\lim _{n \uparrow+\infty} N_{0, n}$ satisfait au principe de domination. La suite $\left(N_{0, n}\right)_{n=1}^{\infty}$ étant décroissante, $\lim _{n \uparrow+\infty} N_{0, n}$ est régulier. On a, pour tout $V$ de $\mathscr{V}_{0}$ et pour tout $n \geqq 1, N_{n}-\eta_{n, C V} \geqq \frac{1}{n} \varepsilon$. De la même manière que ci-dessus (cf. 
le lemme 1); $N_{0, n}$ est un noyau de convolution de Hunt sur $X$, et on obtient ensuite que $N_{n}^{\prime}$ est un noyau de convolution singulier sur $X$ pseudo-périodique à tout le point de $\operatorname{supp}\left(N_{0, n}\right)$. Donc $\lim _{n \uparrow+\infty} N_{n}^{\prime}$ est un noyau de convolution sur $X$ satisfaisant au principe de domination et pseudo-périodique à tout le point de $\operatorname{supp}\left(\lim _{n \uparrow+\infty} N_{0, n}\right)$. Evidemment $\lim _{n \uparrow+\infty} N_{n}^{\prime}$ est singulier, et on a $N=\lim _{n \uparrow+\infty} N_{0, n}+\lim _{n \uparrow+\infty} N_{n}^{\prime}$. Posons $N_{0, \infty}=\lim _{n \uparrow+\infty} N_{0, n}$ et $N_{\infty}^{\prime}=\lim _{n \uparrow+\infty} N_{n}^{\prime}$. On peut supposer $N_{0, \infty} \neq 0$ et $N_{\infty}^{\prime} \neq 0$. Alors il existe une fonction positive, continue et exponentielle $\psi$ sur $X$ telle que $\psi N_{\infty}^{\prime}$ soit périodique à tout le point de $\operatorname{supp}\left(N_{0, \infty}\right)$. Alors, de la même manière que dans (3) de la démonstration du présent théorème, $\psi N_{0, \infty}$ est borné. Posons

$$
\Gamma=\left\{x \in X ;\left(\psi N_{0, \infty}\right)=\left(\psi N_{0, \infty}\right) * \varepsilon_{x}\right\} ;
$$

alors $\Gamma$ est un sous-groupe fermé de $X$. D'après la régularité de $\psi N_{0, \infty}$, $\Gamma$ set compact, et par suite la restreinte de $\psi$ sur $\Gamma$ est égale à 1 . Donc $N$ est périodique à tout le point de $\Gamma$. Il existe de noyaux de convolution $\bar{N}_{0}$ et $\bar{N}^{\prime}$ sur $X / \Gamma$ tels que $\psi N_{0, \infty}$ et $\psi N_{\infty}^{\prime}$ soient respectivement les prolongements canoniques de $\bar{N}_{0}$ et de $\bar{N}^{\prime}$ sur $X$. Il est facile de voir que $\bar{N}_{0}$ est un noyau de convolution borné et régulier sur $X / \Gamma$ et que $\bar{N}^{\prime}$ est un noyau de convolution singulier sur $X$ périodique à tout le point de $\operatorname{supp}\left(\bar{N}_{0}\right) . \quad \bar{N}_{0}$ est non-périodique, et par suite, d'après le lemme $1, \bar{N}_{0}$ est un noyau de convolution borné de Hunt sur $X / \Gamma$. On obtient aussi que $\psi$ est le prolongement canonique sur $X$ d'une fonction positive, continue et exponentielle $\bar{\psi}$ dans $X / \Gamma$. Posons $\bar{\varphi}=1 / \bar{\psi}$; alors $N$ est le prolongement canonique de $\bar{\varphi}\left(\bar{N}_{0}+\bar{N}^{\prime}\right)$ sur $X$. La démonstration est ainsi complète.

Les deux corollaires suivants en résultent immédiatement.

COROLlaIRE 5. Soit $N$ un noyau de convolution non-périodique sur $X$. Pour que $N$ satisfasse au principe de domination, il faut et il suffit que $N$ soit un noyau de convolution de Hunt sur $X$ ou bien que $N$ soit de la forme

$$
N=\varphi\left(\widetilde{N}_{0}+\widetilde{N^{\prime}}\right)
$$

où $\varphi$ est une fonction positive, continue et exponentielle sur $X, \widetilde{N}_{0}$ est un noyau de convolution borné de Hunt sur $X$ ou bien égal à 0 et où $\widetilde{N^{\prime}}$ est un noyau de convolution singulier $(\neq 0)$ sur $X$ périodique à tout le point de $\operatorname{supp}\left(\widetilde{N}_{0}\right)$. 
CoRollaire 6. Soit $N$ un noyau de convolution sur $X$ tel que le sous-groupe fermé de $X$ constitué par toutes les périodes de $N$ ne contienne aucun sous-groupe compact excepté $\{0\}$. Alors la même conclusion a lieu.

\section{BIBLIOGRAPHIES}

[1] G. Choquet et J. Deny: Aspects linéaires de la théorie du potentiel. Noyaux de composition satisfaisant au principe du balayage sur tout ouvert. C. R. Acad. Sci. Paris, 250 (1960), p. 4260-4262.

[2] J. Deny: Sur l'équation de convolution $\mu=\mu * \sigma$ Sém. Brelot-Choquet-Deny, 4e année, $1959 / 60, \mathrm{n}^{\circ} 5$.

[ 3 ] J. Deny: Noyaux de convolution de Hunt et noyaux associés à une famille fondamentale. Ann. Inst. Fourier, 12 (1962), p. 643-667.

[4] M. Itô: Sur les principes divers du maximum et le type positif. Nagoya Math. J., 44 (1971), p. 133-164.

[5] M. Itô: Sur le principe de domination pour les noyaux de convolution. Nagoya Math. J., 50 (1973), p. 149-173.

[6] M. Itô: Sur la famille sous-ordonnée au noyau de convolution de Hunt II. Nagoya Math. J., 53 (1974), p. 115-126.

[7] M. Itô: Une caractérisation du principe de domination pour les noyaux de convolution. Nagoya Math. J., à paraître.

[ 8] M. Itô: Sur le principe relatif de domination pour les noyaux de convolution. Hiroshima Math. J., à paraître.

[9] M. Itô: Remarque sur la définition du principe relatif de domination. Proc. Japan Acad., à paraître.

Université de Nagoya 
